\title{
The last refuge of mixed wino-Higgsino dark matter
}

\author{
M. Beneke, ${ }^{a}$ A. Bharucha, ${ }^{b}$ A. Hryczuk, ${ }^{c, d}$ S. Recksiegel ${ }^{a}$ and P. Ruiz-Femenía ${ }^{a, e}$ \\ ${ }^{a}$ Physik Department T31, Technische Universität München, \\ James-Franck-Straße 1, D-85748 Garching, Germany \\ ${ }^{b}$ Aix Marseille Univ, Université de Toulon, CNRS, CPT, \\ Marseille, France \\ ${ }^{c}$ Department of Physics, University of Oslo, \\ Box 1048, NO-0371 Oslo, Norway \\ ${ }^{d}$ National Centre for Nuclear Research, \\ Hoża 69, 00-681, Warsaw, Poland \\ e Departamento de Fúsica Teórica and Instituto de Fúsica Teórica UAM-CSIC, \\ Universidad Autónoma de Madrid, \\ E-28049 Madrid, Spain \\ E-mail: aoife.bharucha@cpt.univ-mrs.fr, a.j.hryczuk@fys.uio.no, \\ stefan.recksiegel@tum.de, pedro.ruiz-femenia@tum.de
}

ABSTRACT: We delineate the allowed parameter and mass range for a wino-like dark matter particle containing some Higgsino admixture in the MSSM by analysing the constraints from diffuse gamma-rays from the dwarf spheroidal galaxies, galactic cosmic rays, direct detection and cosmic microwave background anisotropies. A complete calculation of the Sommerfeld effect for the mixed-neutralino case is performed. We find that the combination of direct and indirect searches poses significant restrictions on the thermally produced winoHiggsino dark matter with correct relic density. For $\mu>0$ nearly the entire parameter space considered is excluded, while for $\mu<0$ a substantial region is still allowed, provided conservative assumptions on astrophysical uncertainties are adopted.

KEYWORDS: Supersymmetry Phenomenology

ARXIV EPRINT: 1611.00804 


\section{Contents}

1 Introduction 1

2 CR fluxes from wino-like dark matter 3

2.1 Dominantly-wino DM with thermal relic density in the MSSM 3

2.2 Branching fractions and primary spectra 4

3 Indirect and direct searches $\quad 8$

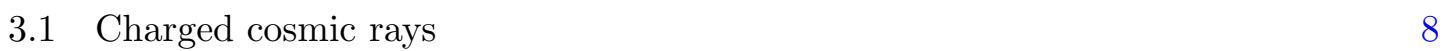

$\begin{array}{lll}3.1 .1 & \text { Propagation } & 8\end{array}$

$\begin{array}{ll}3.1 .2 & \text { Background models }\end{array}$

$\begin{array}{lll}3.2 & \text { Diffuse gamma-rays from dSphs } & 10\end{array}$

$\begin{array}{lll}3.3 & \text { CMB constraints } & 11\end{array}$

$\begin{array}{lll}3.4 & \text { Direct detection } & 11\end{array}$

4 Results: indirect detection and CMB limits $\quad 14$

$\begin{array}{lll}4.1 & \text { Limits on mixed-wino DM } & 14\end{array}$

$\begin{array}{lll}4.2 & \text { Indirect search constraints on the MSSM parameter space } & 16\end{array}$

$\begin{array}{lll}4.3 & \text { Limits on the present-day cross section for fixed }|\mu|-M_{2} & 18\end{array}$

5 Results: including direct detection limits $\quad 19$

$\begin{array}{ll}5.1 \mu>0 & 21\end{array}$

$\begin{array}{ll}5.2 \mu<0 & 21\end{array}$

6 Conclusions 23

\section{Introduction}

Many remaining regions in the parameter space of the Minimal Supersymmetric Standard Model (MSSM), which yield the observed thermal relic density for neutralino dark matter, rely on very specific mechanisms, such as Higgs-resonant annihilation in the so-called funnel region, or sfermion co-annihilation. In [1] we identified new regions, where the dark matter particle is a mixed - as opposed to pure - wino, has mass in the $\mathrm{TeV}$ region, and yields the observed relic density. These new regions are driven to the correct relic abundance by the proximity of the resonance of the Sommerfeld effect due to electroweak gauge boson exchange. In such situations, the annihilation cross section is strongly velocity dependent, and the present-day annihilation cross section is expected to be relatively large, potentially leading to observable signals in indirect searches for dark matter (DM). On the other hand, a substantial Higgsino fraction of a mixed dark matter particle leads to a large, potentially observable dark matter-nucleon scattering cross section. 
In this paper we address the question of which part of this region survives the combination of direct and indirect detection constraints. For the latter we consider diffuse gamma-rays from the dwarf spheroidal galaxies (dSphs), galactic cosmic rays (CRs) and cosmic microwave background (CMB) anisotropies. These have been found to be the most promising channels for detecting or excluding the pure-wino DM model [2]. Stronger limits can be obtained only from the non-observation of the gamma-line feature and to a lesser extent from diffuse gamma-rays both originating in the Galactic Centre (GC). Indeed, it has been shown $[3,4]$ that the pure-wino model is ruled out by the absence of an excess in these search channels, unless the galactic dark matter profile develops a core, which remains a possibility. Since the viability of wino-like DM is a question of fundamental importance, we generally adopt the weaker constraint in case of uncertainty, and hence we take the point of view that wino-like DM is presently not excluded by gamma-line and galactic diffuse gamma-ray searches. Future results from the Črenkov Telescope Array (CTA) are expected to be sensitive enough to resolve this issue (see e.g. [5, 6]), and will either observe an excess in gamma-rays or exclude the dominantly wino DM MSSM parameter region discussed in the present paper.

Imposing the observed relic density as a constraint, the pure-wino DM model has no free parameters and corresponds to the limit of the MSSM when all other superpartner particles and non-standard Higgs bosons are decoupled. Departing from the pure wino in the MSSM introduces many additional dimensions in the MSSM parameter space and changes the present-day annihilation cross section, branching ratios (BRs) for particular primary final states, and the final gamma and CR spectra leading to a modification of the limits. The tools for the precise computation of neutralino dark matter (co-) annihilation in the generic MSSM when the Sommerfeld enhancement is operative have been developed in [7-9] and applied to relic density computations in $[1,10]$. The present analysis is based on an extension of the code to calculate the annihilation cross sections for all exclusive two-body final states separately, rather than the inclusive cross section.

Further motivation for the present study is provided by the spectrum of the cosmic antiproton-to-proton ratio reported by the AMS-02 collaboration [11], which appears to be somewhat harder than expected from the commonly adopted cosmic-ray propagation models. In [12] it has been shown that pure-wino DM can improve the description of this data. Although our understanding of the background is insufficient to claim the existence of a dark matter signal in antiprotons, it is nevertheless interesting to check whether the surviving mixed-wino DM regions are compatible with antiproton data.

The outline of this paper is as follows. In section 2 we summarize the theoretical input, beginning with a description of the dominantly wino MSSM parameter region satisfying the relic-density constraint, then providing some details on the computation of the DM annihilation rates to primary two-body final states. The following section 3 supplies information about the implementation of the constraints from diffuse gamma-rays from the dSphs, galactic CRs, direct detection and the CMB, and the data employed for the analysis. The results of the indirect detection analysis are presented in section 4 as constraints in the plane of the two most relevant parameters of the MSSM, the wino mass parameter $M_{2}$ and $|\mu|-M_{2}$, where $\mu$ is the Higgsino mass parameter. In section 5 the indirect detection 
constraints are combined with that from the non-observation of dark matter-nucleon scattering. For the case of $\mu<0$ we demonstrate the existence of a mixed wino-Higgsino region satisfying all constraints, while for $\mu>0$ we show that there is essentially no remaining parameter space left. Section 6 concludes.

\section{CR fluxes from wino-like dark matter}

\subsection{Dominantly-wino DM with thermal relic density in the MSSM}

In [1] the Sommerfeld corrections to the relic abundance computation for $\mathrm{TeV}$-scale neutralino dark matter in the full MSSM have been studied. The ability to perform the computations for mixed dark matter at a general MSSM parameter space point [7-10] revealed a large neutralino mass range with the correct thermal relic density, which opens mainly due to the proximity of the resonance of the Sommerfeld effect and its dependence on MSSM parameters. In this subsection we briefly review the dominantly-wino parameter region identified in [1], which will be studied in this paper. "Dominantly-wino" or "winolike" here refers to a general MSSM with non-decoupled Higgs bosons, sfermions, bino and Higgsinos as long as the mixed neutralino dark matter state is mainly wino. We also require that its mass is significantly larger than the electroweak scale. The well-investigated pure-wino model refers to the limit in this parameter space, when all particles other than the triplet wino are decoupled.

Despite the large number of parameters needed to specify a particular MSSM completely, in the dominantly-wino region, the annihilation rates depend strongly only on a subset of parameters. These are the wino, bino and Higgsino mass parameters $M_{2}, M_{1}$ and $\mu$, respectively, which control the neutralino composition and the chargino-neutralino mass difference, and the common sfermion mass parameter $M_{\mathrm{sf}}$. In this work we assume that the bino is much heavier that the wino, that is, the lightest neutralino is a mixed wino-Higgsino. Effectively a value of $\left|M_{1}\right|$ larger than $M_{2}$ by a few $100 \mathrm{GeV}$ is enough to decouple the bino in the $\mathrm{TeV}$ region. ${ }^{1}$ The wino mass parameter determines the lightest neutralino (LSP) mass, and the difference $|\mu|-M_{2}$ the wino-Higgsino admixture. In the range $M_{2}=1-5 \mathrm{TeV}$ considered here, the relation $m_{\mathrm{LSP}} \simeq M_{2}$ remains accurate to a few GeV, when some Higgsino fraction is added to the LSP state, and values of $|\mu|-M_{2} \gtrsim 500 \mathrm{GeV}$ imply practically decoupled Higgsinos.

Increasing the Higgsino component of the wino-like LSP lowers its coupling to charged gauge bosons, to which wino-like neutralinos annihilate predominantly, and therefore increases the relic density. Larger mixings also imply that the mass difference between the lightest chargino and neutralino increases, which generically reduces the size of the Sommerfeld enhancement of the annihilation cross section. These features are apparent in the contours of constant relic density in the $|\mu|-M_{2}$ vs. $M_{2}$ plane for the wino-Higgsino case shown in [1], which are almost straight for large $|\mu|-M_{2}$, but bend to lower values of $m_{\text {LSP }}$ as $|\mu|-M_{2}$ is reduced. A representative case is reproduced in figure 1. The contours

\footnotetext{
${ }^{1}$ Allowing for significant bino admixture leads to other potentially interesting, though smaller regions, as described in [1].
} 
also bend towards lower $M_{2}$ when sfermions become lighter, as they mediate the t- and u-channel annihilation into SM fermions, which interferes destructively with the s-channel annihilation, effectively lowering the co-annihilation cross section. By choosing small values of $M_{\mathrm{sf}}$ (but larger than $1.25 m_{\mathrm{LSP}}$ to prevent sfermion co-annihilation, not treated by the present version of the code), LSP masses as low as $1.7 \mathrm{TeV}$ are seen to give the correct thermal density, to be compared with the pure-wino result, $m_{\mathrm{LSP}} \simeq 2.8 \mathrm{TeV}$.

For $M_{2}>2.2 \mathrm{TeV}$ a resonance in the Sommerfeld-enhanced rates is present, which extends to larger $M_{2}$ values as the Higgsino fraction is increased. The enhancement of the cross section in the vicinity of the resonance makes the contours of constant relic density cluster around it and develop a peak that shifts $m_{\mathrm{LSP}}$ to larger values. In particular, the largest value of $M_{2}$, which gives the correct thermal relic density, is close to $3.3 \mathrm{TeV}$, approximately $20 \%$ higher than for the pure-wino scenario. The influence of the less relevant MSSM Higgs mass parameter $M_{A}$ is also noticeable when the LSP contains some Higgsino admixture, which enhances the couplings to the Higgs (and Z) bosons in s-channel annihilation. This is more pronounced if $M_{A}$ is light enough such that final states containing heavy Higgs bosons are kinematically accessible. The corresponding increase in the annihilation cross section results in positive shifts of around 100 to $250 \mathrm{GeV}$ in the value of $M_{2}$ giving the correct relic density on decreasing $M_{A}$ from $10 \mathrm{TeV}$ to $800 \mathrm{GeV}$. In summary, a large range of lightest neutralino masses, $1.7-3.5 \mathrm{TeV}$, provides the correct relic density for the mixed wino-Higgsino state as a consequence of the Sommerfeld corrections.

The MSSM parameter points considered in this paper have passed standard collider, flavour and theoretical constraints as discussed in [1]. In the dominantly-wino parameter space, most of the collider and flavour constraints are either satisfied automatically or receive MSSM corrections that are suppressed or lie within the experimental and theoretical uncertainties. Ref. [1] further required compatibility with direct dark matter detection constraints by imposing that the DM-nucleon spin-independent cross section was less than twice the LUX limits reported at the time of publication [13]. This did not affect the results significantly, see figure 1, as in most of the parameter space of interest the scattering cross section was predicted to be much above those limits. ${ }^{2}$ Recently the LUX collaboration has presented a new limit, stronger than the previous one by approximately a factor of four [14], potentially imposing more severe constraints on the dominantly-wino neutralino region of the MSSM parameter space. The details of the implementation of the limits from indirect detection searches for the mixed wino, which were not included in our previous analysis, and from the new LUX results are given in section 3.

\subsection{Branching fractions and primary spectra}

The annihilation of wino-like DM produces highly energetic particles, which subsequently decay, fragment and hadronize into stable SM particles, producing the CR fluxes.

The primary particles can be any of the SM particles, and the heavy MSSM Higgs bosons, $H^{0}, A^{0}$ and $H^{ \pm}$, when they are kinematically accessible. We consider neutralino

\footnotetext{
${ }^{2}$ The irregularities in the contours in this and the following figures are artifacts of the interpolation. In particular, in figure 1 the relic density contours corresponding to $\Omega h^{2}=0.06$ are in reality connected vertically.
} 


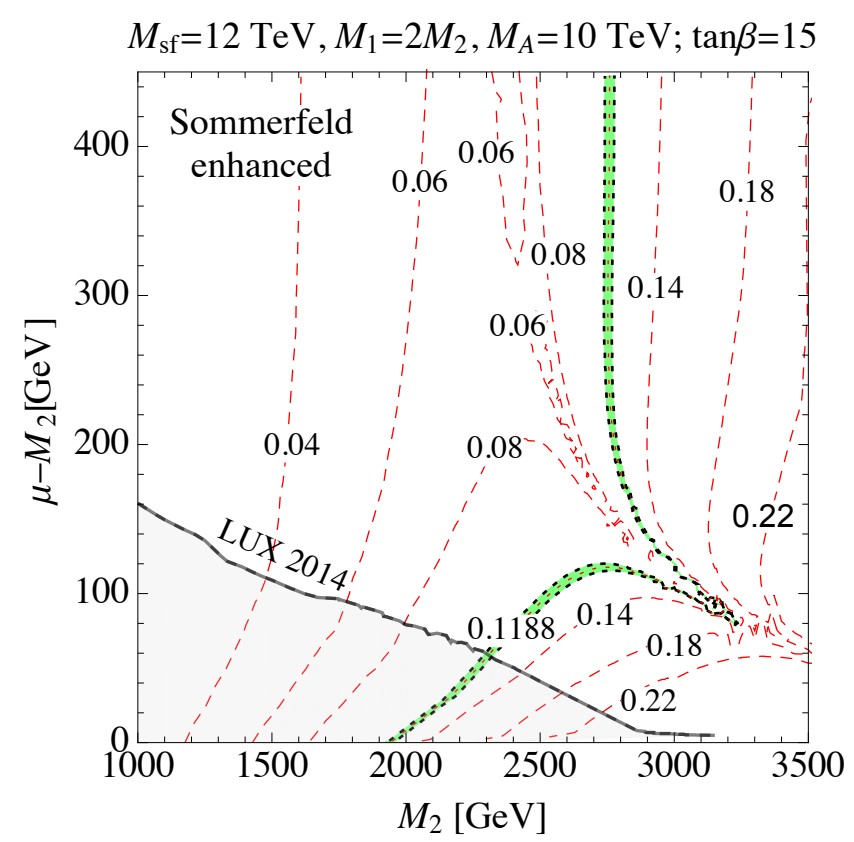

Figure 1. Contours of constant relic density in the $M_{2}$ vs. $\left(\mu-M_{2}\right)$ plane for $\mu>0$, as computed in [1]. The (green) band indicates the region within $2 \sigma$ of the observed dark matter abundance. Parameters are as given in the header, and the trilinear couplings are set to $A_{i}=8 \mathrm{TeV}$ for all sfermions except for that of the stop, which is fixed by the Higgs mass value. The black solid line corresponds to the old LUX limit [13] on the spin-independent DM-nucleon cross section, which excludes the shaded area below this line. Relaxing the old LUX limit by a factor of two to account for theoretical uncertainties eliminates the direct detection constraint on the shown parameter space region.

dark matter annihilation into two primary particles. The number of such exclusive twobody channels is 31 , and the corresponding neutralino annihilation cross sections are computed including Sommerfeld loop corrections to the annihilation amplitude as described in $[1,7,9]$. As input for this calculation we need to provide the tree-level exclusive annihilation rates of all neutral neutralino and chargino pairs, since through Sommerfeld corrections the initial LSP-LSP state can make transitions to other virtual states with heavier neutralinos or a pair of conjugated charginos, which subsequently annihilate into the primaries. The neutralino and chargino tree-level annihilation rates in the MSSM have been derived analytically in [7], and including $v^{2}$-corrections in [8], in the form of matrices, where the off-diagonal entries refer to the interference of the short-distance annihilation amplitudes of different neutralino/chargino two-particle states into the same final state. For the present analysis the annihilation matrices have been generalized to vectors of matrices, such that the components of the vector refer to the 31 exclusive final states. The large number of different exclusive final states can be implemented without an increase in the CPU time for the computation relative to the inclusive case. Since the information about the exclusive annihilation rates only enters through the (short-distance) annihilation matrices, the two-particle wave-functions that account for the (long-distance) Sommerfeld 
corrections only need to be computed once. On the contrary, since the $v^{2}$-corrections to the annihilation of DM in the present Universe are very small, they can be neglected, which results in a significant reduction in the time needed to compute the annihilation matrices. ${ }^{3}$ It further suffices to compute the present-day annihilation cross section for a single dark matter velocity, and we choose $v=10^{-3} c$. The reason for this choice is that the Sommerfeld effect saturates for very small velocities, and the velocity dependence is negligible for velocities smaller than $10^{-3} \mathrm{c}$. In other words, while the dark matter velocities follow distributions centered around $v=10^{-4}-10^{-5} c$ for dwarf galaxies and around $10^{-3} c$ for our galaxy, this is irrelevant for the computation.

The energy spectrum $d N_{f} / d x$ of a stable particle $f$ at production per DM annihilation can be written as

$$
\frac{d N_{f}}{d x}=\sum_{I} \operatorname{Br}_{I} \frac{d N_{I \rightarrow f}}{d x}
$$

where $x=E_{f} / m_{\mathrm{LSP}}$, and $d N_{I \rightarrow f} / d x$ represents the contribution from each two-body primary final state $I$ with branching fraction $\mathrm{Br}_{I}$ to the spectrum of $f$ after the decay, fragmentation and hadronization processes have taken place. We compute $\mathrm{Br}_{I}$ from our MSSM Sommerfeld code as described above and use the tables for $d N_{I \rightarrow f} / d x$ provided with the PPPC4DMID code [15], which include the leading logarithmic electroweak corrections through the electroweak fragmentation functions [16].

Two comments regarding the use of the spectra provided by the PPPC4DMID code are in order. The code only considers primary pairs $I$ of a particle together with its antiparticle, both assumed to have the same energy spectrum. For wino-like DM there exist primary final states with different species, i.e. $I=i j$ with $j \neq \bar{i}$, such as $Z \gamma$ and $Z h^{0}$. In this case, we compute the final number of particles $f$ produced from that channel as one half of the sum of those produced by channels $I=i \bar{i}$ and $I=j \bar{j}$. This is justified, since the fragmentation of particles $i$ and $j$ is independent. A second caveat concerns the heavy MSSM Higgs bosons that can be produced for sufficiently heavy neutralinos. These are not considered to be primary channels in the PPPC4DMID code, which only deals with SM particles. A proper treatment of these primaries would first account for the decay modes of the heavy Higgs bosons, and then consider the fragmentation and hadronization of the SM multi-particle final state in an event generator. Instead of a full treatment, we replace the charged Higgs $H^{ \pm}$by a longitudinal-polarized $W^{ \pm}$-boson, and the neutral heavy Higgses $H^{0}, A^{0}$ by the light Higgs $h^{0}$ when computing the spectra in $x$. This approximation is not very well justified. However, the branching ratios of the dominantly-wino neutralino to final states with heavy Higgses are strongly suppressed, and we could equally have set them to zero without a noticeable effect on our results.

The branching fractions of primary final states obtained from our code are shown in the left panel of figure 2 as a function of the Higgsino fraction for a wino-like LSP with $2 \mathrm{TeV}$ mass. The pure wino annihilates mostly to $W^{+} W^{-}$and to a lesser extent to other pairs of gauge bosons, including the loop-induced photon final state, which is generated by

\footnotetext{
${ }^{3}$ Since we also computed the relic density for every parameter point, which requires including the $v^{2}-$ corrections, we did not make use of this simplification in the present analysis.
} 

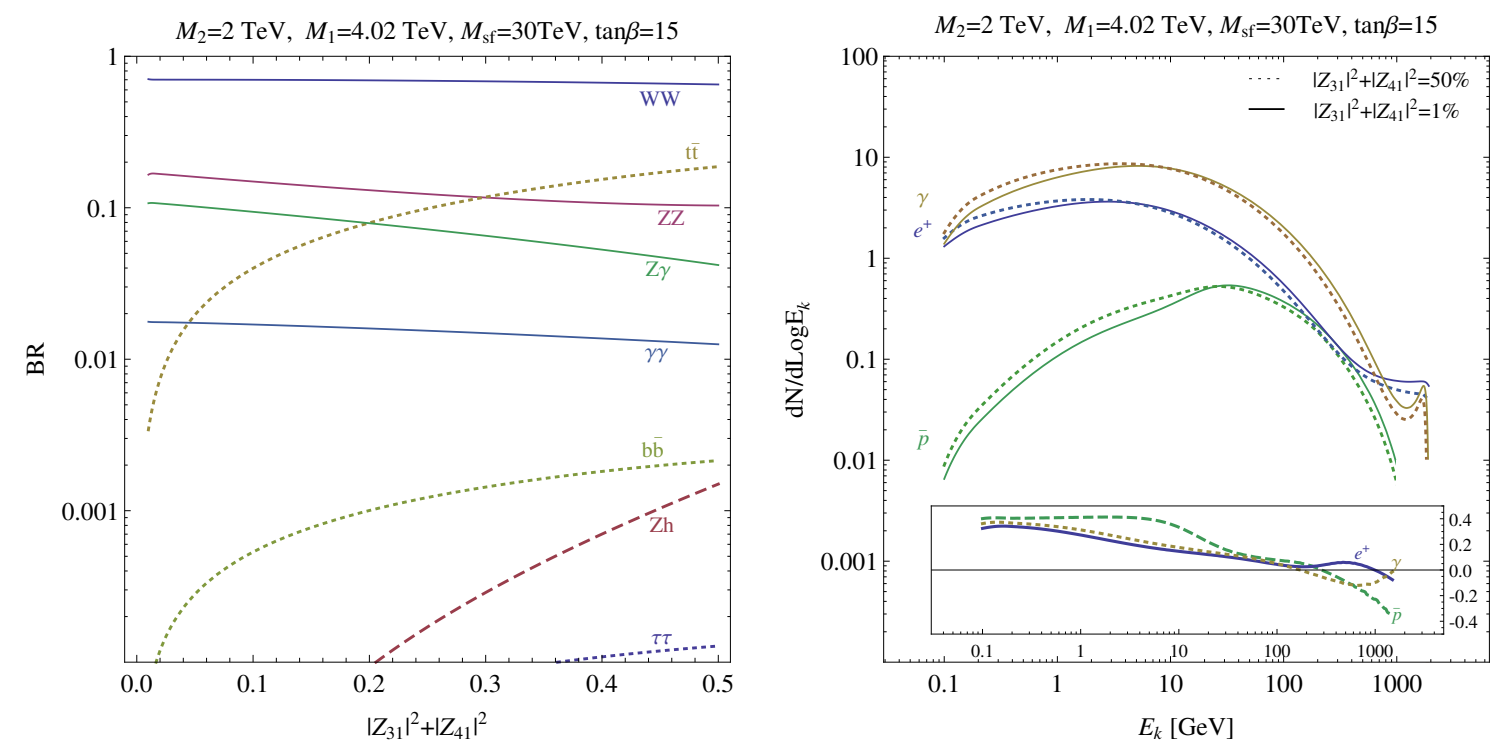

Figure 2. Left: branching fractions of present-day wino-like neutralino annihilation vs. the Higgsino fraction for decoupled $M_{A}$ and sfermions. $\left|Z_{31}\right|^{2}+\left|Z_{41}\right|^{2}$ refers to the Higgsino fraction of the lightest neutralino in the convention of [7]. Right: comparison of $\bar{p}, e^{+}$and gamma-ray spectra per annihilation at production of a $50 \%$ mixed wino-Higgsino (dashed) to the pure-wino (solid) model. The gamma-line component is not shown. In the inset at the bottom of the plot the relative differences between the two spectra are shown.

the Sommerfeld correction. The annihilation to fermions is helicity or $p$-wave suppressed. The suppression is lifted only for the $t \bar{t}$ final state as the Higgsino admixture increases, in which case this final state becomes the second most important. Except for this channel, the dominant branching fractions are largely independent of the Higgsino fraction. The annihilation to $W^{+} W^{-}$is always dominant and above $75 \%$.

The final spectra of photons, positrons and antiprotons per annihilation at production for small (solid lines) and large (dashed lines) Higgsino mixing are plotted in the right panel of figure 2. The spectra in these two extreme cases are very similar, because $W^{+} W^{-}$is the dominant primary final state largely independent of the wino-Higgsino composition, and also the number of final stable particles produced by the sub-dominant primary channels do not differ significantly from each other. The inset in the right-hand plot shows that the relative change between the mixed and pure wino case varies from about $+40 \%$ to about $-40 \%$ over the considered energy range. Concerning the variation with respect to the DM mass, the most important change is in the total annihilation cross section, not in the spectra $d N_{f} / d x$. The branching ratios $\operatorname{Br}_{I}$ to primaries depend on the LSP mass in the $\mathrm{TeV}$ regime only through the Sommerfeld corrections, which can change the relative size of the different channels. However, since for wino-like neutralinos annihilation into $W^{+} W^{-}$dominates the sum over $I$ in $(2.1)$, the dependence of the final spectra on $m_{\mathrm{LSP}}$ is very mild. 


\section{Indirect and direct searches}

In this section we discuss our strategy for determining the constraints on mixed-wino dark matter from various indirect searches. While the analysis follows that for the pure wino [2], here we focus on the most relevant search channels: the diffuse gamma-ray emission from dSphs, antiprotons and positron CRs, and the CMB. Moreover, since we consider winolike DM with a possibly significant Higgsino admixture, we implement the direct detection constraints as well.

\subsection{Charged cosmic rays}

\subsubsection{Propagation}

The propagation of charged CRs in the Galaxy is best described within the diffusion model with possible inclusion of convection. In this framework the general propagation equation takes the form [17]

$$
\begin{aligned}
& \frac{\partial N^{i}}{\partial t}-\vec{\nabla} \cdot\left(D_{x x} \vec{\nabla}-\vec{v}_{c}\right) N^{i}+\frac{\partial}{\partial p}\left(\dot{p}-\frac{p}{3} \vec{\nabla} \cdot \overrightarrow{v_{c}}\right) N^{i}-\frac{\partial}{\partial p} p^{2} D_{p p} \frac{\partial}{\partial p} \frac{N^{i}}{p^{2}} \\
& =Q^{i}(p, r, z)+\sum_{j>i} c \beta n_{\text {gas }}(r, z) \sigma_{i j} N^{j}-c \beta n_{\text {gas }}(r, z) \sigma_{\mathrm{in}} N^{i}-\sum_{j<i} \frac{N^{i}}{\tau^{i \rightarrow j}}+\sum_{j>i} \frac{N^{j}}{\tau^{j \rightarrow i}},
\end{aligned}
$$

where $N^{i}(p, r, z)$ is the number density of the $i$-th particle species with momentum $p$ and corresponding velocity $v=c \beta$, written in cylindrical coordinates $(r, z), \sigma_{\text {in }}$ the inelastic scattering cross section, $\sigma_{i j}$ the production cross section of species $i$ by the fragmentation of species $j$, and $\tau^{i \rightarrow j}, \tau^{j \rightarrow i}$ are the lifetimes related to decays of $i$ and production from heavier species $j$, respectively.

We solve (3.1) with the help of the DRAGON code [18], assuming cylindrical symmetry and no convection, $\vec{v}_{c}=0$. With the galacto-centric radius $r$, the height from the Galactic disk $z$ and rigidity $R=p c / Z e$, we adopt the following form of the spatial diffusion coefficient:

$$
D_{x x}(R, r, z)=D_{0} \beta^{\eta}\left(\frac{R}{R_{0}}\right)^{\delta} e^{|z| / z_{d}} e^{\left(r-r_{\odot}\right) / r_{d}} .
$$

The momentum-space diffusion coefficient, also referred to as reaccelaration, is related to it via $D_{p p} D_{x x}=p^{2} v_{A}^{2} / 9$, where the Alfvén velocity $v_{A}$ represents the characteristic velocity of a magnetohydrodynamic wave. The free parameters are the normalization $D_{0}$, the spectral indices $\eta$ and $\delta$, the parameters setting the radial scale $r_{d}$ and thickness $z_{d}$ of the diffusion zone, and finally $v_{A}$. We fix the normalization at $R_{0}=3 \mathrm{GV}$. The diffusion coefficient is assumed to grow with $r$, as the large scale galactic magnetic field gets weaker far away from the galactic center.

The source term is assumed to have the form

$$
Q^{i}(R, r, z)=f^{i}(r, z)\left(\frac{R}{R^{i}}\right)^{-\gamma^{i}}
$$

where $f^{i}(r, z)$ parametrizes the spatial distribution of supernova remnants normalized at $R^{i}$, and $\gamma^{i}$ is the injection spectral index for species $i$. For protons and Helium we modify 


\begin{tabular}{|cc|cccc|cc|cc|}
\hline \multicolumn{6}{|c|}{ Benchmark } & \multicolumn{5}{|c|}{ Diffusion } & \multicolumn{4}{|c|}{ Injection } \\
\hline Model & $\begin{array}{c}z_{d} \\
{[\mathrm{kpc}]}\end{array}$ & $\delta$ & $\begin{array}{c}D_{0} / 10^{28} \\
{\left[\mathrm{~cm}^{2}{ }^{-1}\right]}\end{array}$ & $\begin{array}{c}v_{A} \\
{\left[\mathrm{~km} \mathrm{~s}^{-1}\right]}\end{array}$ & $\eta$ & $\gamma_{1}^{p} / \gamma_{2}^{p} / \gamma_{3}^{p}$ & $\begin{array}{c}R_{0,1}^{p} \\
\mathrm{GV}\end{array}$ & $\gamma_{1}^{\mathrm{He}} / \gamma_{2}^{\mathrm{He}} / \gamma_{3}^{\mathrm{He}}$ & $\begin{array}{c}R_{0,1}^{\mathrm{He}} \\
\mathrm{GV}\end{array}$ \\
\hline Thin & 1 & 0.47 & 0.43 & 13.0 & -0.37 & $1.85 / 2.39 / 2.22$ & 6.8 & $2.18 / 2.35 / 2.16$ & 12.7 \\
\hline Med & 4 & 0.5 & 1.79 & 14.0 & -0.3 & $1.90 / 2.36 / 2.21$ & 7.7 & $2.18 / 2.35 / 2.16$ & 12.7 \\
\hline Thick & 10 & 0.5 & 3.3 & 14.5 & -0.27 & $2.00 / 2.38 / 2.22$ & 7.5 & $2.18 / 2.36 / 2.12$ & 12.7 \\
\hline
\end{tabular}

Table 1. Benchmark propagation models. The radial length is always $r_{d}=20 \mathrm{kpc}$ and convection is neglected, $\vec{v}_{c}=0$. The second break in the proton injection spectra is at $300 \mathrm{GV}$. For primary electrons we use a broken power-law with spectral indices 1.6/2.65 and a break at $7 \mathrm{GV}$, while for heavier nuclei we assumed one power-law with index 2.25. $R_{0,1}^{i}$ refer to the positions of the first and second break, respectively, and $\gamma_{1,2,3}^{i}$ to the power-law in the three regions separated by the two breaks. The propagation parameters were obtained by fitting to $\mathrm{B} / \mathrm{C}$, proton and He data and cross-checked with antiproton data, while the primary electrons were obtained from the measured electron flux.

the source term to accommodate for two breaks in the power-law, as strongly indicated by observations. Leptons lose energy very efficiently, thus those which are very energetic need to be very local, while we do not observe nor expect many local sources of $\mathrm{TeV}$ scale leptons. This motivates multiplying (3.3) by an additional exponential cut-off in energy, $e^{-E / E_{c}}$, with $E_{c}$ set to $50 \mathrm{TeV}$ for electron and positron injection spectra.

We employ the gas distribution $n_{\text {gas }}$ derived in [19, 20] and adopt the standard forcefield approximation [21] to describe the effect of solar modulation. The modulation potential is assumed to be a free parameter of the fit and is allowed to be different for different CR species.

\subsubsection{Background models}

In [2] 11 benchmark propagation models with varying diffusion zone thickness, from $z_{d}=1$ $\mathrm{kpc}$ to $z_{d}=20 \mathrm{kpc}$, were identified by fitting to the B/C, proton, Helium, electron and $e^{+}+e^{-}$data. Since then the AMS-02 experiment provided CR spectra with unprecedented precision, which necessitates modifications of the above benchmark models. Following the same procedure as in [2] we choose three representative models, which give a reasonable fit to the AMS-02 data, denoted Thin, Med and Thick, corresponding to the previous $z_{d}=1 \mathrm{kpc}, z_{d}=4 \mathrm{kpc}$ and $z_{d}=10 \mathrm{kpc}$ models. ${ }^{4}$ The relevant parameters are given in table 1. In figure 3 we show the fit to the B/C and the AMS-02 proton data [23-25] and superimpose the older data from PAMELA [26, 27]. In all these cases, as well as for the lepton data $[28,29]$, the measurements used in the fits were from AMS-02 results only.

\footnotetext{
${ }^{4}$ We loosely follow here the widely adopted MIN, MED, MAX philosophy [22], choosing models with as large variation in the DM-originated antiproton flux as possible. However, the MIN, MED, MAX models were optimized for pre-AMS data and are based on a semi-analytic diffusion model. Since we rely on the full numerical solution of the diffusion equation, we follow the benchmark models of [2]. This comes at the expense of no guarantee that the chosen models really provide the minimal and maximal number of antiprotons. However, as in this work we are not interested in setting precise limits from antiproton data, we consider this approach as adequate.
} 

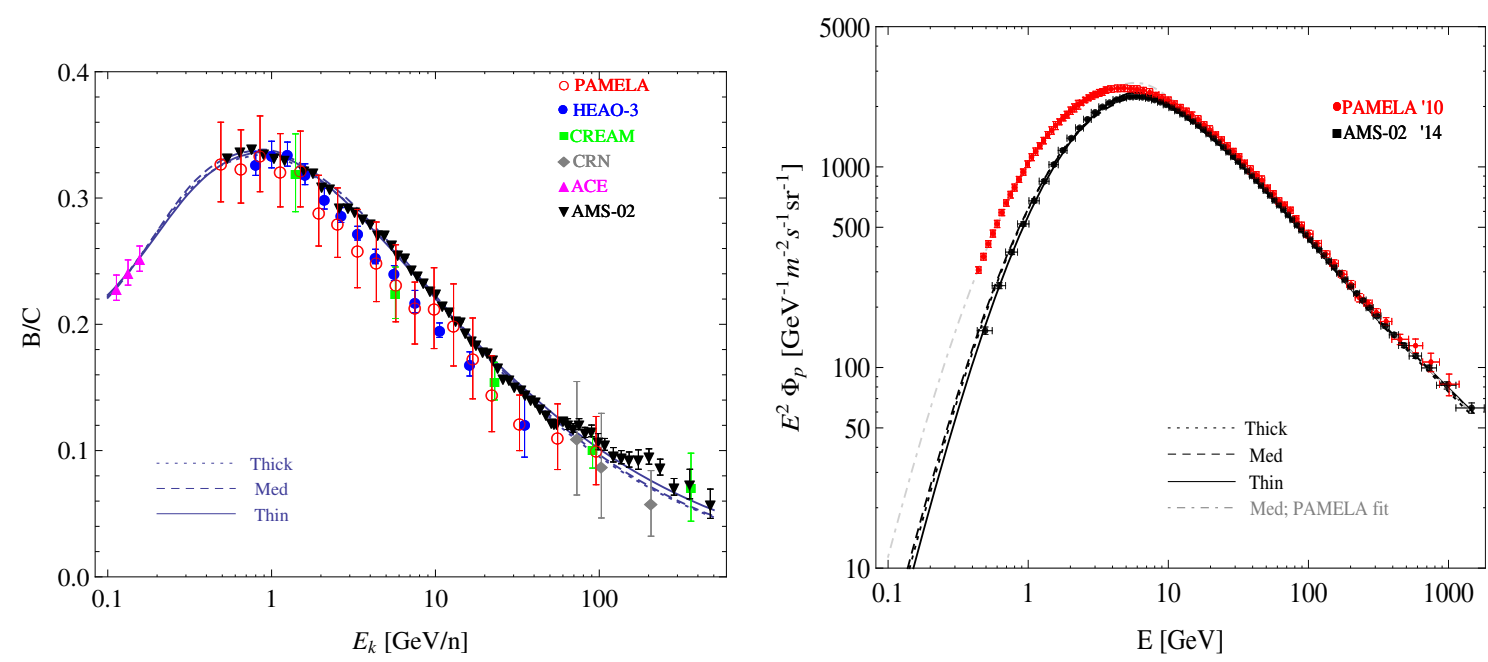

Figure 3. Comparison of the benchmark propagation models: B/C (left) and protons (right). The fit was performed exclusively to the AMS-02 [23-25] measurements, while the other data sets are shown only for comparison: PAMELA [26, 27], HEAO-3 [30], CREAM [31], CRN [32], ACE [33].

In the fit we additionally assumed that the normalization of the secondary CR antiprotons can freely vary by $10 \%$ with respect to the result given by the DRAGON code. This is motivated by the uncertainty in the antiproton production cross sections. The impact of this and other uncertainties has been studied in detail in e.g. [34-36].

As we will show below, the DM contribution to the lepton spectra is of much less importance for constraining the parameter space of our interest, therefore, we do not discuss the lepton backgrounds explicitly. All the details of the implementation of the lepton limits closely follow [2], updated to the published AMS-02 data [28, 29].

\subsection{Diffuse gamma-rays from dSphs}

Recently the Fermi-LAT and MAGIC collaborations released limits from the combination of their stacked analyses of 15 dwarf spheroidal galaxies [37]. Here we use the results of this analysis to constrain the parameter space of the mixed wino-Higgsino neutralino. To this end we compute all exclusive annihilation cross sections for present-day DM annihilation in the halo and take a weighted average of the limits provided by the experimental collaborations. As discussed in section 2.2, the $\mathrm{TeV}$ scale wino-like neutralino annihilates predominantly into $W^{+} W^{-}, Z Z$ and $t \bar{t}$, with much smaller rates into leptons and the lighter quarks. In the results from [37] only the $W^{+} W^{-}, b \bar{b}, \mu^{+} \mu^{-}$and $\tau^{+} \tau^{-}$final states are given. However, as the predicted spectrum and number of photons from a single annihilation is not significantly different for the hadronic or leptonic final states, we adopt the approximation that the limits from annihilation into $Z Z$ are the same as from $W^{+} W^{-}$, while those from $t \bar{t}$ and $c \bar{c}$ are the same as $b \bar{b}$. The differences in the number of photons produced between these annihilation channels in the relevant energy range are maximally of order $\mathcal{O}(20 \%)$ for $W^{+} W^{-}$vs. $Z Z$ and $t \bar{t}$ vs. $b \bar{b}$. Comparing $b \bar{b}$ to light quarks these can rise up to factor 2 , however due to helicity suppression these channels have negligible 
branching fractions. Hence, the adopted approximation is expected to be very good and, the corresponding uncertainty is significantly smaller than that related to the astrophysical properties of the dSphs (parametrised by the $J$-factors).

\subsection{CMB constraints}

The annihilation of dark matter at times around recombination can affect the recombination history of the Universe by injecting energy into the pre-recombination photon-baryon plasma and into the post-recombination gas and background radiation, which has consequences for the power and polarization spectra of the CMB [38-40]. In particular, it can result in the attenuation of the temperature and polarization power spectra, more so on smaller scales, and in a shift of the TE and EE peaks. These effects can be traced back to the increased ionization fraction and baryon temperature, resulting in a broadening of the surface of last scattering, which suppresses perturbations on scales less than the width of this surface. Therefore the CMB temperature and polarization angular power spectra can be used to infer upper bounds on the annihilation cross section of dark matter into a certain final state for a given mass. When Majorana dark matter particles annihilate, the rate at which energy $E$ is released per unit volume $V$ can be written as

$$
\frac{d E}{d t d V}(z)=\rho_{\text {crit }}^{2} \Omega^{2}(1+z)^{6} p_{\text {ann }}(z)
$$

where $\rho_{\text {crit }}$ is the critical density of the Universe today, and experiment provides constraints on $p_{\text {ann }}(z)$, which describes the effects of the DM. These effects are found to be well enough accounted for when the $z$ dependence of $p_{\text {ann }}(z)$ is neglected, such that a limit is obtained for the constant $p_{\text {ann }}$. The latest $95 \%$ C.L. upper limit on $p_{\text {ann }}$ was obtained by Planck [41], and we adopt their most significant limit $3.4 \cdot 10^{-28} \mathrm{~cm}^{3} \mathrm{~s}^{-1} \mathrm{GeV}^{-1}$ from the combination of TT, TE, EE + lowP + lensing data. The constant $p_{\text {ann }}$ can further be expressed via

$$
p_{\text {ann }}=\frac{1}{M_{\chi}} f_{\text {eff }}\langle\sigma v\rangle,
$$

where $f_{\text {eff }}$, parametrizing the fraction of the rest mass energy that is injected into the plasma or gas, must then be calculated in order to extract bounds on the DM annihilation cross section in the recombination era. In our analysis, for $f_{\text {eff }}$ we use the quantities $f_{\text {eff,new }}^{I}$ from [42] for a given primary annihilation channel $I$. We then extract the upper limit on the annihilation cross section at the time of recombination by performing a weighted average over the contributing annihilation channels, as done for the indirect detection limits discussed in section 3.2. As the Sommerfeld effect saturates before this time, $\langle\sigma v\rangle$ at recombination is the same as the present-day cross section. In the future the cross section bound can be improved by almost an order of magnitude, until $p_{\text {ann }}$ is ultimately limited by cosmic variance.

\subsection{Direct detection}

Direct detection experiments probe the interaction of the dark matter particle with nucleons. For the parameter space of interest here, the bounds on spin-independent interactions, 
sensitive to the t-channel exchange of the Higgs bosons and to s-channel sfermion exchange are more constraining than those on spin-dependent interactions. The coupling of the lightest neutralino to a Higgs boson requires both a Higgsino and gaugino component, and is therefore dependent on the mixing. Note that the relative size of the Higgs Yukawa couplings means that the contribution due to the Higgs coupling to strange quarks dominates the result.

In the pure-wino limit, when the sfermions are decoupled and the coupling to the Higgs bosons vanishes, the direct detection constraints are very weak as the elastic scattering takes place only at the loop level [43]. Allowing for a Higgsino admixture and/or non-decoupled sfermions introduces tree-level scattering processes mediated by Higgs or sfermion exchange. Direct detection experiments have recently reached the sensitivity needed to measure such low scattering cross sections and with the new data released by the LUX [14] and PandaX [44] collaborations, a portion of the discussed parameter space is now being probed.

In the analysis below we adopt the LUX limits [14], being the strongest in the neutralino mass range we consider. In order to be conservative, in addition to the limit presented by the collaboration we consider a weaker limit by multiplying by a factor of two. This factor two takes into account the two dominant uncertainties affecting the spin-independent cross section, i.e. the local relic density of dark matter and the strange quark content of the nucleon. The former, $\rho=0.3 \pm 0.1 \mathrm{GeV} / \mathrm{cm}^{3}$, results in an uncertainty of $50 \%$ [45] and the latter result contributes an uncertainty on the cross section of about $20 \%$ [46], which on combination result in weakening the bounds by a factor of two (denoted as $\times 2$ on the plots). For the computation of the spin-independent scattering cross section for every model point we use micrOMEGAs [47, 48]. Note that the Sommerfeld effect does not influence this computation and the tree-level result is expected to be accurate enough.

Since only mixed Higgsino-gaugino neutralinos couple to Higgs bosons, the limits are sensitive to the parameters affecting the mixing. To be precise, for the case that the bino is decoupled $\left(\left|M_{1}\right| \gg M_{2},|\mu|\right)$ and $|\mu|-M_{2} \gg m_{Z}$, the couplings of the Higgs bosons $h, H$ to the lightest neutralino are proportional to

$$
c_{h}=m_{Z} c_{W} \frac{M_{2}+\mu \sin 2 \beta}{\mu^{2}-M_{2}^{2}}, \quad c_{H}=-m_{Z} c_{W} \frac{\mu \cos 2 \beta}{\mu^{2}-M_{2}^{2}},
$$

where $c_{W} \equiv \cos \theta_{W}$, and it is further assumed that $M_{A}$ is heavy such that $c_{h, H}$ can be computed in the decoupling limit $\cos (\alpha-\beta) \rightarrow 0$. When $\tan \beta$ increases, the light Higgs coupling $c_{h}$ decreases for $\mu>0$ and increases for $\mu<0$. On the other hand the coupling $c_{H}$ increases in magnitude with $\tan \beta$ for both $\mu>0$ and $\mu<0$, but is positive when $\mu>0$ and negative for $\mu<0$. In addition, in the decoupling limit the coupling of the light Higgs to down-type quarks is SM-like, and the heavy Higgses couple to down-type quarks proportionally to $\tan \beta$. The sfermion contribution is dominated by the gauge coupling of the wino-like component neutralino to the sfermion and the quarks. We remark that for the parameter range under consideration there is destructive interference between the 

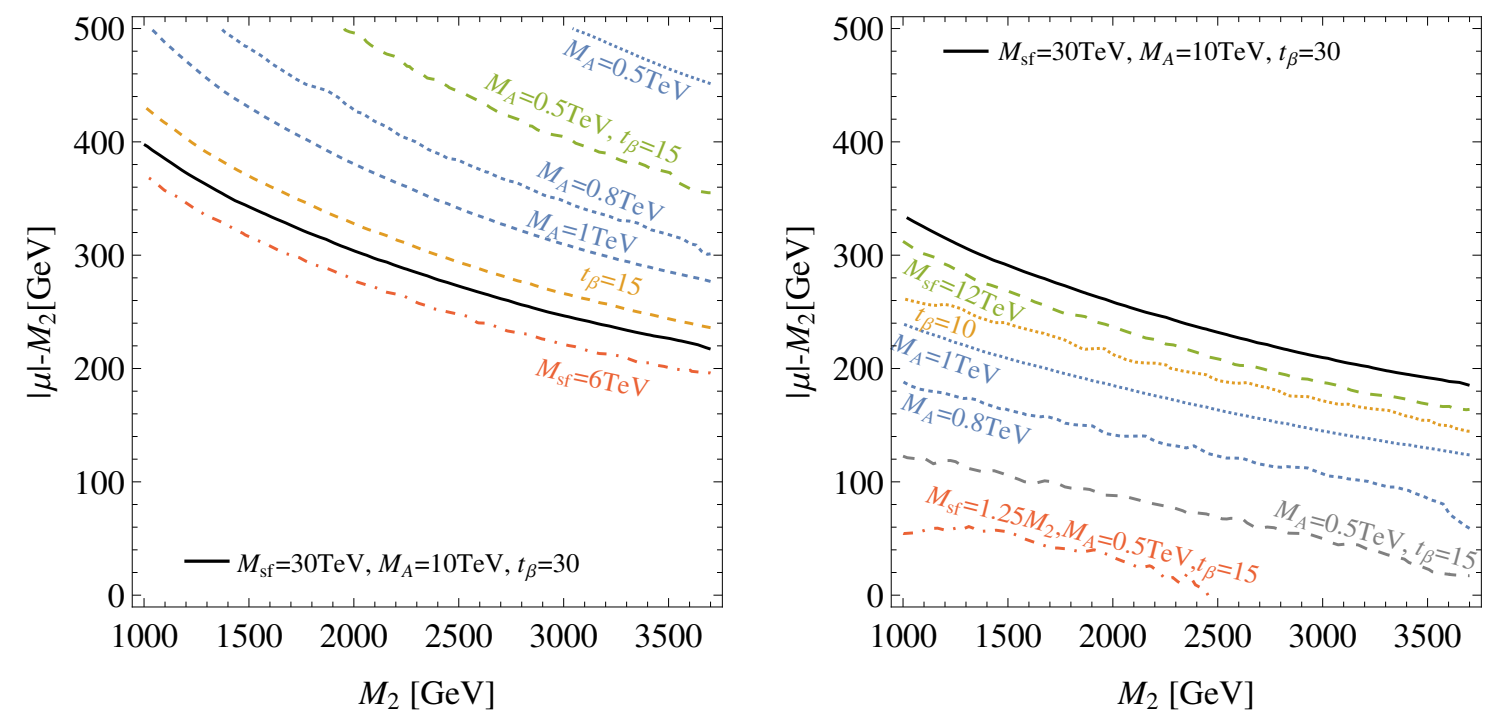

Figure 4. Direct detection limits for different choices of the MSSM parameters, assuming the neutralino is completely responsible for the measured dark matter density of the Universe. Where not stated, the parameter choices correspond to those for the black line. The area below the lines is excluded. The left panel shows the case of $\mu>0$, while the right of $\mu<0$.

amplitude for the Higgs and sfermion-exchange diagrams for $\mu>0$, and for $\mu<0$ when [49]

$$
\frac{m_{H}^{2}\left(1-2 / t_{\beta}\right)}{m_{h}^{2}}<t_{\beta},
$$

provided $M_{2} \simeq|\mu|$ and $t_{\beta} \equiv \tan \beta \gg 1$. For these cases lower values of the sfermion masses reduce the scattering cross section.

In figure 4 we show the resulting limits from LUX data in the $|\mu|-M_{2}$ vs. $M_{2}$ plane for different choices of $t_{\beta}, M_{A}, M_{\mathrm{sf}}$, and the sign of $\mu$. The above discussion allows us to understand the following trends observed:

- On decreasing $t_{\beta}$ and $M_{A}$ the direct detection bound becomes stronger for positive $\mu$ and weaker for negative $\mu$. Note that for $\mu<0$ the cross section decreases, and the bound weakens, due to the destructive interference between the $h$ and $H$ contributions as the relative sign between the couplings $c_{h}$ and $c_{H}$ changes.

- The direct detection bound weakens for less decoupled sfermions when there is destructive interference between the t-channel Higgs-exchange and s-channel sfermionexchange diagrams. This always occurs for $\mu>0$, while for $\mu<0$ one requires small heavy Higgs masses. For instance, for $t_{\beta}=15$ the maximum value of $M_{A}$ giving destructive interference is slightly above $500 \mathrm{GeV}$, while for $t_{\beta}=30$ one needs $M_{A}<700 \mathrm{GeV}$.

Since we consider a point in the $|\mu|-M_{2}$ vs. $M_{2}$ plane to be excluded only if it is excluded for any (allowed) value of the other MSSM parameters, this means that the bounds from direct detection experiments are weakest for $\mu<0$ in combination with low values of $M_{\mathrm{sf}}$, $M_{A}$ and $\tan \beta$, and for $\mu>0$ in combination with high values of $M_{A}$ and $\tan \beta$ but low values of $M_{\mathrm{sf}}$. 


\section{Results: indirect detection and CMB limits}

In this section we first determine the region of the $|\mu|-M_{2}$ vs. $M_{2}$ plane which satisfies the relic density constraint and is allowed by the gamma-ray limits from dwarf spheroidals, the positron limits from AMS-02, and the CMB limits. ${ }^{5}$ We also determine the regions preferred by fits to AMS-02 antiproton results. Over a large part of the considered $|\mu|-M_{2}$ vs. $M_{2}$ plane, the observed relic density can be obtained for some value of the sfermion masses and other MSSM parameters. For the remaining region of the plane, where the relic density constraint is not fulfilled for thermally produced neutralino dark matter, we consider both, the case where the dark matter density is that observed throughout the plane, in which case it cannot be produced thermally, and the case where it is always thermally produced, for which the neutralino relic density does not always agree with that observed, and the limits must be rescaled for each point in the plane by the relic density calculated accordingly. That the neutralino dark matter is not thermally produced, or that it only constitutes a part of the total dark matter density are both viable possibilities.

We then consider various slices through this plane for fixed values of $|\mu|-M_{2}$, and show the calculated present-day annihilation cross section as a function of $M_{2} \sim m_{\chi_{1}^{0}}$ together with the same limits and preferred regions as above, both for the case that the limits are and are not rescaled according to the thermal relic density.

\subsection{Limits on mixed-wino DM}

In this section we present our results on the limits from indirect searches for wino-like DM in the MSSM, assuming the relic density is as observed. That is, for most parameter points the DM must be produced non-thermally or an additional mechanism for late entropy production is at play. We show each of the considered indirect search channels separately in the $|\mu|-M_{2}$ vs. $M_{2}$ plane (including both $\mu>0$ and $\mu<0$ ), superimposing on this the contours of the correct relic density for three choices of the sfermion mass. Note that while the indirect detection limits are calculated for $M_{\mathrm{sf}}=8 \mathrm{TeV}$, the effect of the choice of sfermion mass on them is minimal, and therefore we display only the relic density contours for additional values of $M_{\mathrm{sf}}$.

In figure 5 we show the exclusions from dSphs, $e^{+}$, and the CMB separately in the $|\mu|-M_{2}$ vs. $M_{2}$ plane. For the positrons we show two limits, obtained on assuming the Thin and Thick propagation models described in section 3.1.2. We see that the most relevant exclusions come from the diffuse gamma-ray searches from dSphs. Here we show three lines corresponding to the limit on the cross section assuming the Navarro-Frenk-White profile in dSphs, and rescaling this limit up and down by a factor 2 . This is done in order to estimate the effect of the uncertainty in the $J$-factors. For instance, the recent reassessment [50] of the $J$-factor for Ursa Minor inferred from observational data suggests 2 to 4 times smaller limits than those commonly quoted. In order to provide conservative bounds, we

\footnotetext{
${ }^{5}$ For the combined $e^{+}+e^{-}$flux several earlier observations provide data extending to higher energies than the AMS-02 experiment, though with much larger uncertainties. We do not include these data in our analysis, because for the DM models under consideration, the strongest lepton limits arise from energies below about $100 \mathrm{GeV}$, in particular from the observed positron fraction (see figure 7 of [2]).
} 

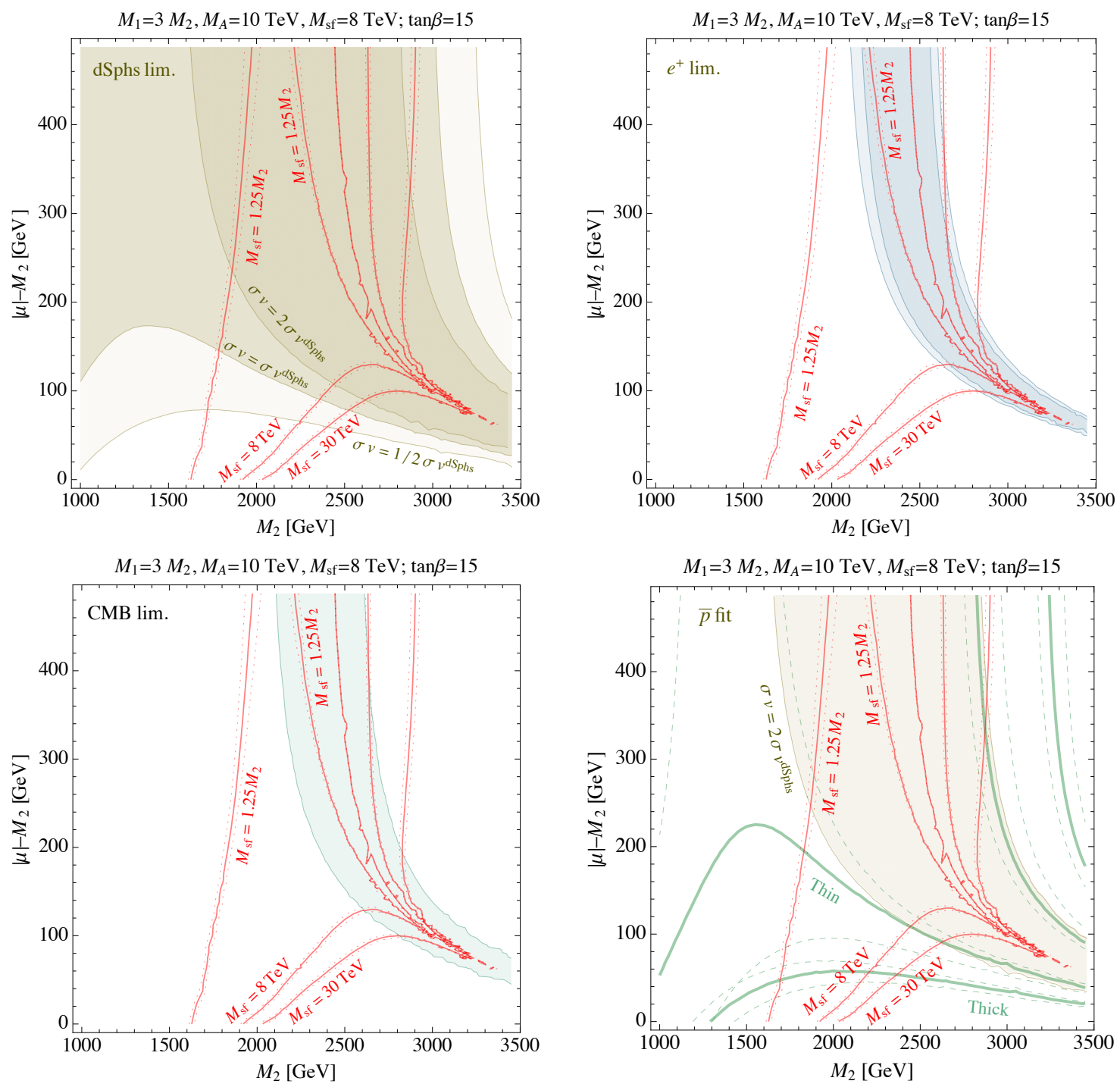

Figure 5. Results in the $M_{2}$ vs. $|\mu|-M_{2}$ plane. Left: limits from dSphs (upper) and the CMB (lower). The shaded regions are excluded, different shadings correspond to the DM profile uncertainty. Right: the region excluded by AMS-02 leptons (upper), and the best fit contours for antiprotons (lower), where the green solid lines show the Thin and Thick propagation models, while the dotted lines around them denote the $1 \sigma$ confidence intervals. Contours where the observed relic density is obtained for the indicated value of the sfermion mass are overlaid.

adopt the weakest of the three as the reference limit. We then compare (lower right plot) this weakest limit from dSphs to the preferred region obtained on fitting to the AMS-02 antiproton results, showing the results for both Thin and Thick propagation models. ${ }^{6}$

We find that there are parts of the mixed wino-Higgsino and dominantly wino neutralino parameter space both below and above the Sommerfeld resonance region, where the

\footnotetext{
${ }^{6}$ The actual analysis was finalized before the recent antiproton results were published [11] and hence was based on earlier data presented by the AMS collaboration [51]. This is expected to have a small effect on the antiproton fit presented in this work, with no significant consequences for the overall results.
} 

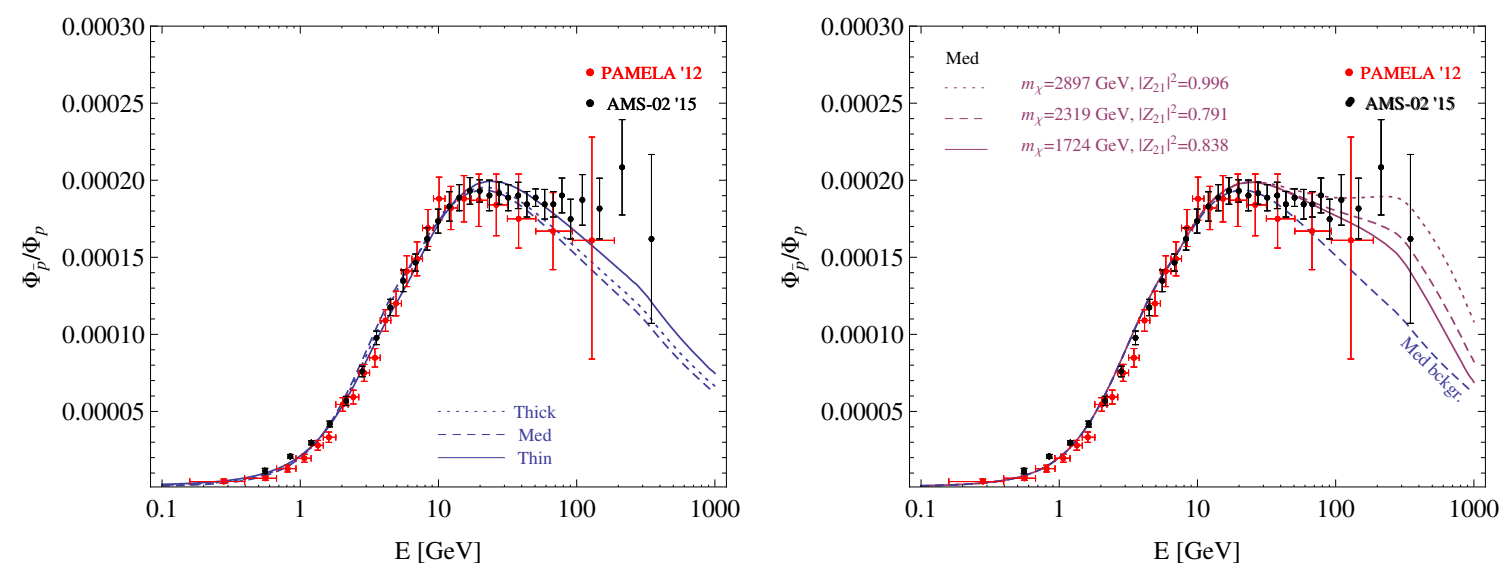

Figure 6. The antiproton-to-proton ratio: background propagation models (left) and comparison of three DM models with relic density within the observational range and assuming the "Med" propagation (right). The shown data is from AMS-02 [51] and PAMELA [52].

relic density is as observed and which are compatible with the non-observation of dark matter signals in indirect searches. In the lower right plot of figure 5 we see that these further overlap with the regions preferred by fits to the antiproton results. In the smaller region above the resonance, this overlap occurs when the sfermions are decoupled, and hence corresponds to an almost pure-wino case, whereas below the resonance the overlap region is spanned by varying the sfermion masses from $1.25 M_{2}$ to being decoupled. The latter region requires substantial Higgsino-mixing of the wino, and extends from $M_{2}=1.7 \mathrm{TeV}$ to about $2.5 \mathrm{TeV}$, thus allowing dominantly-wino dark matter in a significant mass range.

Let us comment on the improvement of the fit to the antiproton measurements found for some choices of the parameters. In figure 6 we show examples of antiproton-to-proton ratio fits to the data from the background models (left) and including the DM component (right). Although the propagation and antiproton production uncertainties can easily resolve the apparent discrepancy of the background models vs. the observed data [34-36], it is nevertheless interesting to observe that the spectral shape of the DM component matches the observed data for viable mixed-wino dark matter particles.

\subsection{Indirect search constraints on the MSSM parameter space}

In this section we present our results for the limits from indirect searches on wino-like DM, assuming the relic density is always thermally produced. In other words, for the standard cosmological model, these constitute the limits on the parameter space of the MSSM, since even if the neutralino does not account for all of the dark matter, its thermal population can give large enough signals to be seen in indirect searches. In this case a parameter-space point is excluded, if

$$
\left.(\sigma v)_{0}\right|_{\text {th }}>\left.\left(\frac{\left.\Omega h^{2}\right|_{\text {obs }}}{\left.\Omega h^{2}\right|_{\text {thermal }}}\right)^{2}(\sigma v)_{0}\right|_{\text {exp lim }}
$$

where $\left.(\sigma v)_{0}\right|_{\text {th }}$ is the theoretically predicted present-day cross section and $\left.(\sigma v)_{0}\right|_{\exp \lim }$ the limit quoted by the experiment. This is because the results presented by the experiments 

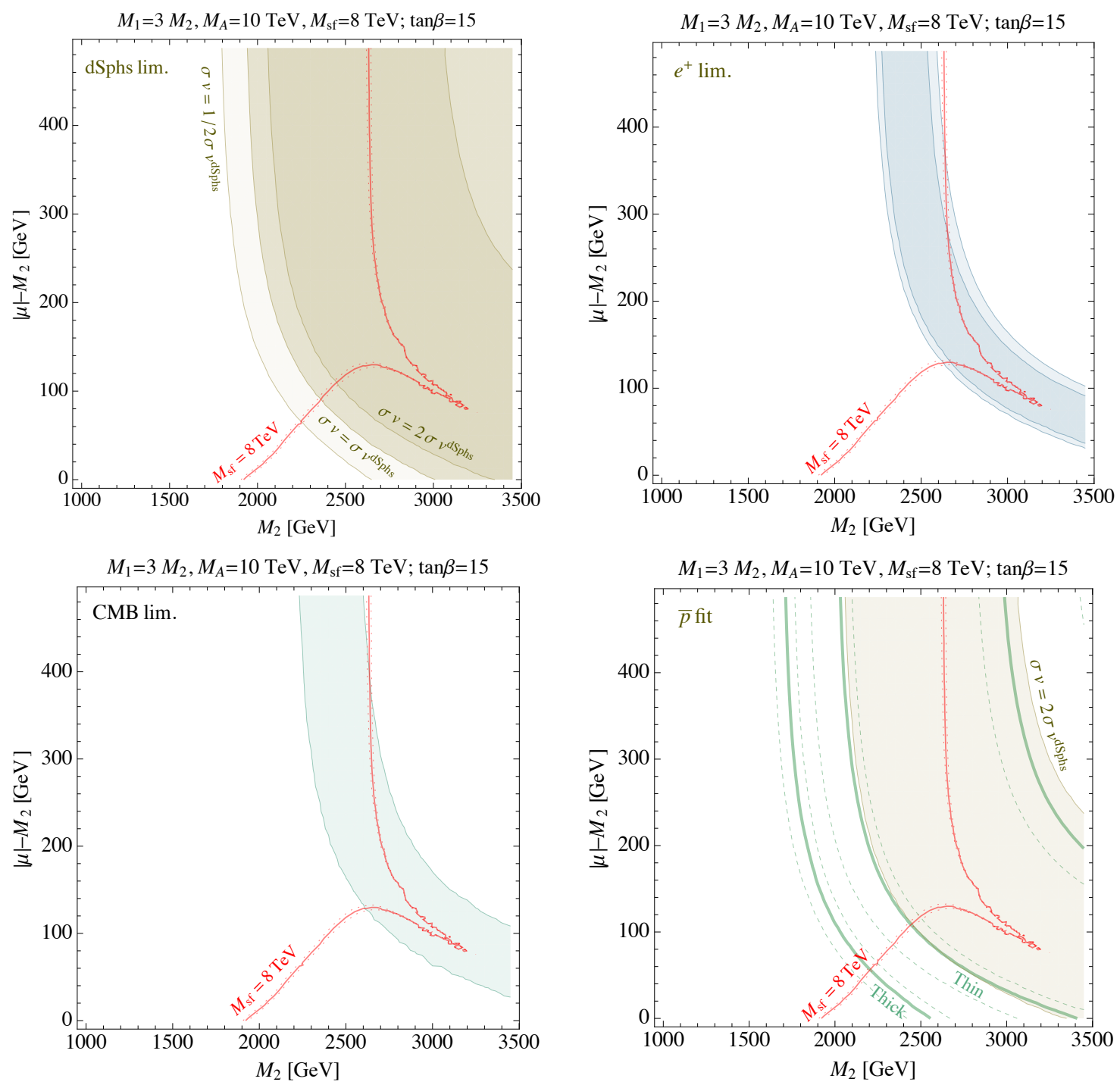

Figure 7. Results in the $M_{2}$ vs $|\mu|-M_{2}$ plane for the case where the limits are rescaled according to the thermal relic density for a given point in the plane. Details are as in figure 5.

assume the DM particle to account for the entire observed relic density. Therefore if one wishes to calculate the limits for dark matter candidates which only account for a fraction of the relic density, one needs to rescale the bounds by the square of the ratio of observed relic density $\left.\Omega h^{2}\right|_{\text {obs }}$ to the thermal relic density $\left.\Omega h^{2}\right|_{\text {thermal }}$. Viewed from another perspective, the results below constitute astrophysical limits on a part of the MSSM parameter space, which is currently inaccessible to collider experiments, with the only assumption that there was no significant entropy production in the early Universe after the DM freeze-out.

In figure 7 , as in the previous subsection, we show the exclusions from dSphs, $e^{+}$, and the CMB individually in the $|\mu|-M_{2}$ vs. $M_{2}$ plane. The limits are calculated as for figure 5 . We then compare the weakest limit from dSphs to the preferred region obtained on fitting to the AMS-02 antiproton results, where we show the results for both Thin and Thick 
propagation models. Again we find that parameter regions exist where the relic density is correct and which are not excluded by indirect searches. The marked difference between the previous and present results is that in figure 7 the region of the plots for lower $M_{2}$ is not constrained by the indirect searches, because in this region the thermal relic density is well below the measured value and therefore the searches for relic neutralinos are much less sensitive. In the bottom lower plot of figure 7 we see that the unconstrained regions overlap with the regions preferred by fits to the antiproton results. While the limits themselves do not depend on the sfermion mass, the thermal relic density does, and therefore the rescaling of the limits via (4.1) induces a dependence on the sfermion mass. Therefore the intersection of the lines of correct relic density for $M_{\mathrm{sf}} \neq 8 \mathrm{TeV}$ with the preferred region from antiproton searches is not meaningful, and we do not show them in the plots.

\subsection{Limits on the present-day cross section for fixed $|\mu|-M_{2}$}

In order to understand how the limits and the present-day annihilation cross section depend on the mass of the DM candidate, we take slices of the $|\mu|-M_{2}$ vs. $M_{2}$ plane for fixed values of $|\mu|-M_{2}$, and plot $(\sigma v)_{0}$ (black) as a function of $M_{2}$, which is approximately equal to the LSP mass $m_{\chi_{1}^{0}}$ in the range shown in figures 8 and 9 . As in figures 5 and 7 we show the limits from dSphs (brown), positrons (blue dashed) and the CMB (magenta dotdashed), along with the preferred regions from antiproton searches (pale green) adopting the Thin and Thick propagation models. We consider three choices of $\mu-M_{2}$ : a very mixed neutralino LSP, $|\mu|-M_{2}=50 \mathrm{GeV}$ where $\mu$ is negative, a mixed case $|\mu|-M_{2}=220 \mathrm{GeV}$ where $\mu$ is positive, and an almost pure-wino scenario, $|\mu|-M_{2}=1000 \mathrm{GeV}$. The blue shaded region indicates where the relic density can correspond to the observed value by changing $M_{\mathrm{sf}}$.

For figure 8 we adopt the unrescaled limit, that is, two sections of figure 5 . In the case of the very mixed wino-Higgsino shown in the upper panel there is a wide range of neutralino masses for which the black curve lies below the conservative dSphs limit and simultaneously within the range of correct relic density spanned by the variation of the sfermion mass. This is different for the almost pure-wino scenario shown in the lower panel, where only a small mass region survives the requirement that the conservative dSphs limit is respected and the observed relic density is predicted. Moreover, in this mass region the sfermions must be almost decoupled. Figure 9 shows two cases of mixed wino-Higgsino dark matter, which exhibit similar features, but now for the case of assumed thermal relic density, such that the limits are rescaled.

It is evident from both figures that for lower values of $|\mu|-M_{2}$, larger regions in $M_{2}$ can provide both the correct relic density and present-day cross section below the dSphs bounds. We also see that while the correct relic density can be attained at the Sommerfeld resonance, the mass regions compatible with indirect search constraints typically lie below the Sommerfeld resonance, as was evident from figures 5 and 7 . 

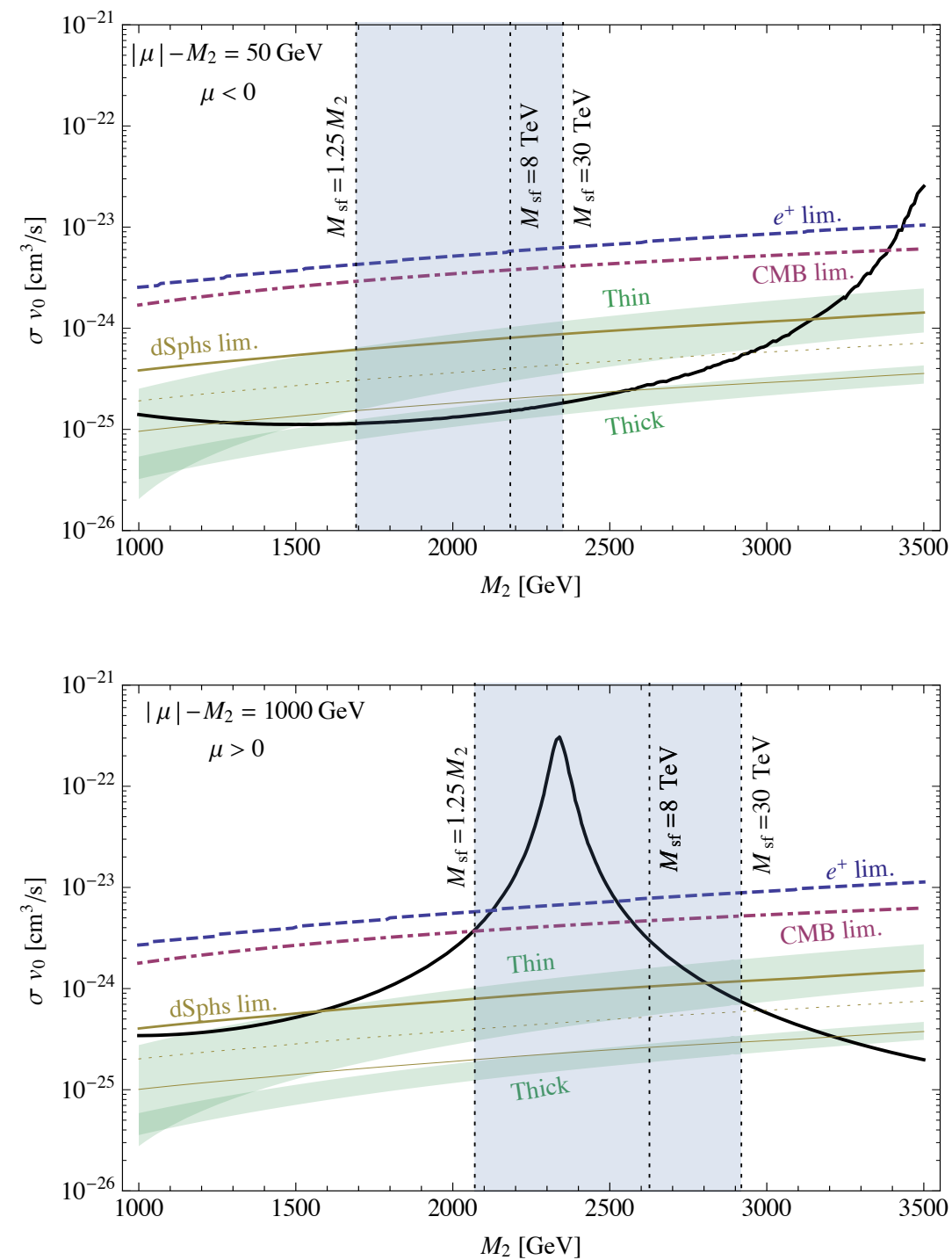

Figure 8. The predicted present-day annihilation cross section $(\sigma v)_{0}$ (black) is shown as a function of $M_{2} \sim m_{\chi_{1}^{0}}$ for the Higgsino admixture $|\mu|-M_{2}$ as indicated. This is compared with exclusion limits from dSphs (brown), positrons (blue dashed) and the CMB (magenta dot-dashed), along with the preferred regions from antiproton searches (pale green) adopting the Thin and Thick models. We also show the dSphs exclusion limits multiplied and divided by 2 (brown), the weaker of which is the thicker line. The observed relic density is assumed. The blue shaded region indicates where the relic density can correspond to the observed value by changing $M_{\mathrm{sf}}$.

\section{Results: including direct detection limits}

We have seen in the previous section that there is a sizeable mixed wino-Higgsino MSSM parameter space where the lightest neutralino has the correct relic abundance and evades indirect detection constraints. A significant Higgsino fraction might, however, be in conflict with the absence of a direct detection signal. In this section we therefore combine the exclusion limits from indirect searches studied in the previous section with those coming from 

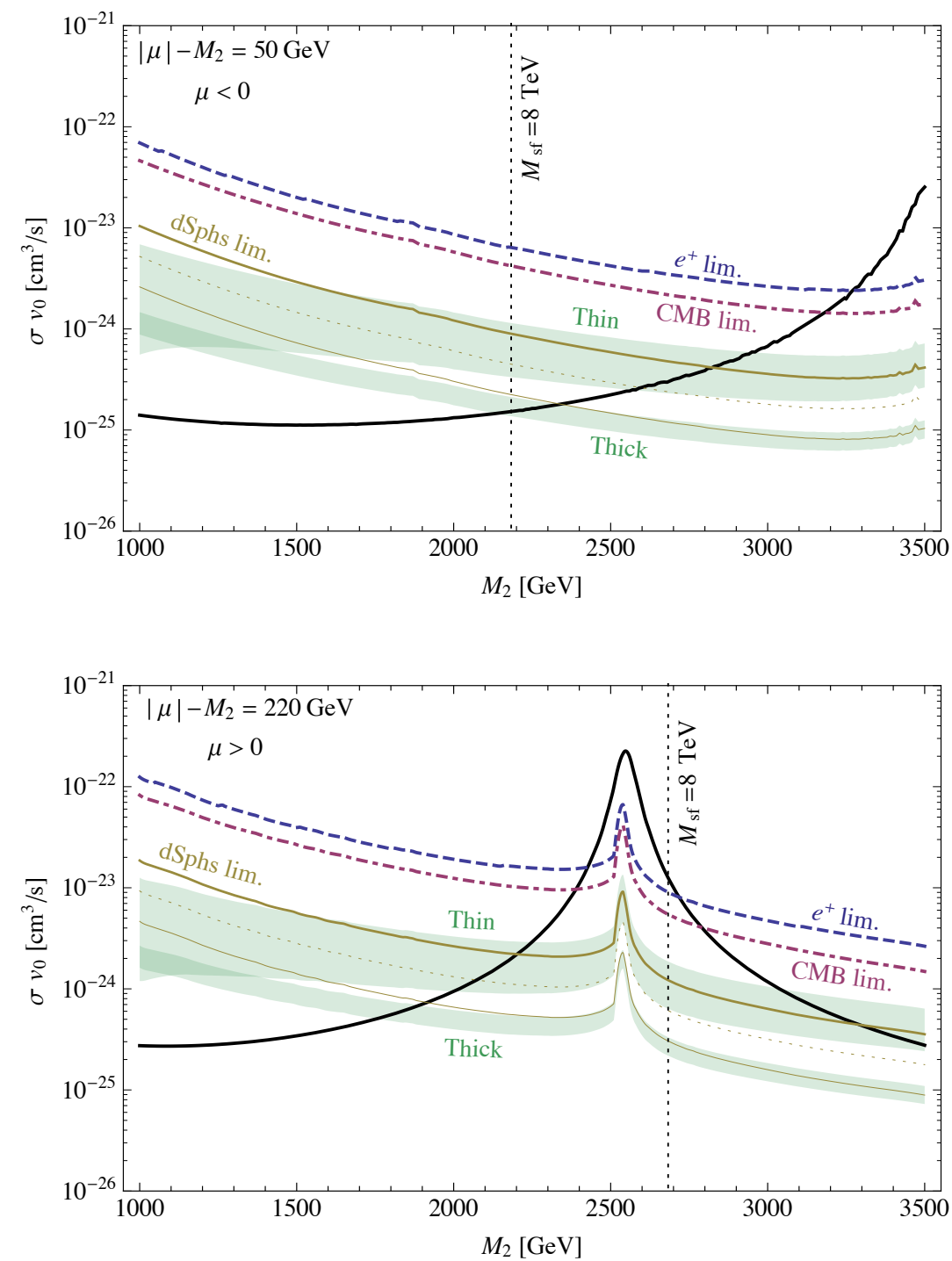

Figure 9. As in figure 8, but the thermal relic density is assumed and the limits are rescaled according to (4.1). Note the different value of $|\mu|-M_{2}$ in the lower plot compared to the previous figure. The black-dashed vertical line indicates where the relic density is equal to that observed for the sfermion mass value $M_{\mathrm{sf}}=8 \mathrm{TeV}$.

the latest LUX results for direct detection, in order to determine the allowed mixed winoHiggsino or dominantly-wino dark matter parameter space. To this end we first determine the maximal region in this space that passes relic density and indirect detection limits in the following way. For a given $|\mu|-M_{2}$ we identify two points in $M_{A}, M_{\mathrm{sf}}$ and $\tan \beta$ within the considered parameter ranges, i.e. $M_{A} \in\{0.5 \mathrm{TeV}, 10 \mathrm{TeV}\}, M_{\mathrm{sf}} \in\left\{1.25 M_{2}, 30 \mathrm{TeV}\right\}$ and $\tan \beta \in\{5,30\},{ }^{7}$ corresponding to maximal and minimal values of $M_{2}$, for which the relic density matches the observed value. Two distinct areas of parameter space arise: the

\footnotetext{
${ }^{7}$ Moving the lower limit $M_{A}=500 \mathrm{GeV}$ to $800 \mathrm{GeV}$ would result in a barely noticeable change to the boundaries marked by $\mathrm{p} 2$.
} 
first is larger and corresponds to a mixed wino-Higgsino whereas the second is narrower and corresponds approximately to the pure wino. The relic density criterion therefore defines one (almost pure wino) or two (mixed wino-Higgsino) sides of the two shaded regions, shown in figures 10 and 11, corresponding to the pure and mixed wino. The dSphs limit defines the other side in the almost pure-wino region, while the remaining sides of the mixed wino-Higgsino area are determined by the dSphs limit (upper), the condition $|\mu|-M_{2}=0$, and the antiproton search (the arc on the lower side of the mixed region beginning at $M_{2} \simeq 1.9 \mathrm{TeV}$ ). We recall that we consider the central dSphs limit and those obtained by rescaling up and down by a factor of two; the shading in grey within each region is used to differentiate between these three choices.

Next we consider the exclusion limits in the $M_{2}$ vs. $|\mu|-M_{2}$ plane from the 2016 LUX results, which have been obtained as outlined in section 3.4. As discussed there, the sign of $\mu$ can strongly influence the strength of the direct detection limits and consequently the allowed parameter space for mixed wino-Higgsino DM. We therefore consider the two cases separately.

\section{$5.1 \mu>0$}

Out of the two distinct regions described above, the close-to-pure wino and the mixed wino-Higgsino, only the former survives after imposing the direct detection constraints, see figure 10. If conservative assumptions are adopted for direct detection and dSphs limits a small triangle at the top of the mixed region is still allowed. The fact that the direct detection constraints mainly impact the mixed rather than the pure wino region was discussed in section 3.4, and is understood in that the Higgs bosons only couple to mixed gaugino-Higgsino neutralinos.

Note that the direct detection limits presented on the plot are for the choice of MSSM parameters giving the weakest possible constraints. This is possible because the boundaries of the maximal region allowed by indirect searches do not depend as strongly on the parameters governing the wino-Higgsino mixing as the spin-independent scattering cross section does. The only exceptions are the boundaries of the mixed-wino region, arising from the relic density constraint, which indeed depend strongly on $M_{\mathrm{sf}}$. However, as varying these boundaries does not significantly change the allowed region, since it is mostly in the part excluded by the LUX data, we choose to display the LUX bound for a value of $M_{\mathrm{sf}}$ different from that defining these boundaries. Therefore, all in all, the case of the mixed wino-Higgsino with $\mu>0$ is verging on being excluded by a combination of direct and indirect searches, when imposing that the lightest neutralino accounts for the entire thermally produced dark matter density of the Universe. Note, however, that the small close-to-pure wino region is not affected by direct detection constraints.

\section{$5.2 \mu<0$}

When $\mu<0$ the spin-independent cross section decreases, particularly for smaller values of $\tan \beta$. This allows for parameter choices with small $|\mu|-M_{2}$ giving viable neutralino $\mathrm{DM}$, in agreement with the direct detection constraint. Indeed, for appropriate parameter choices the direct detection limits are too weak to constrain any of the relevant regions 


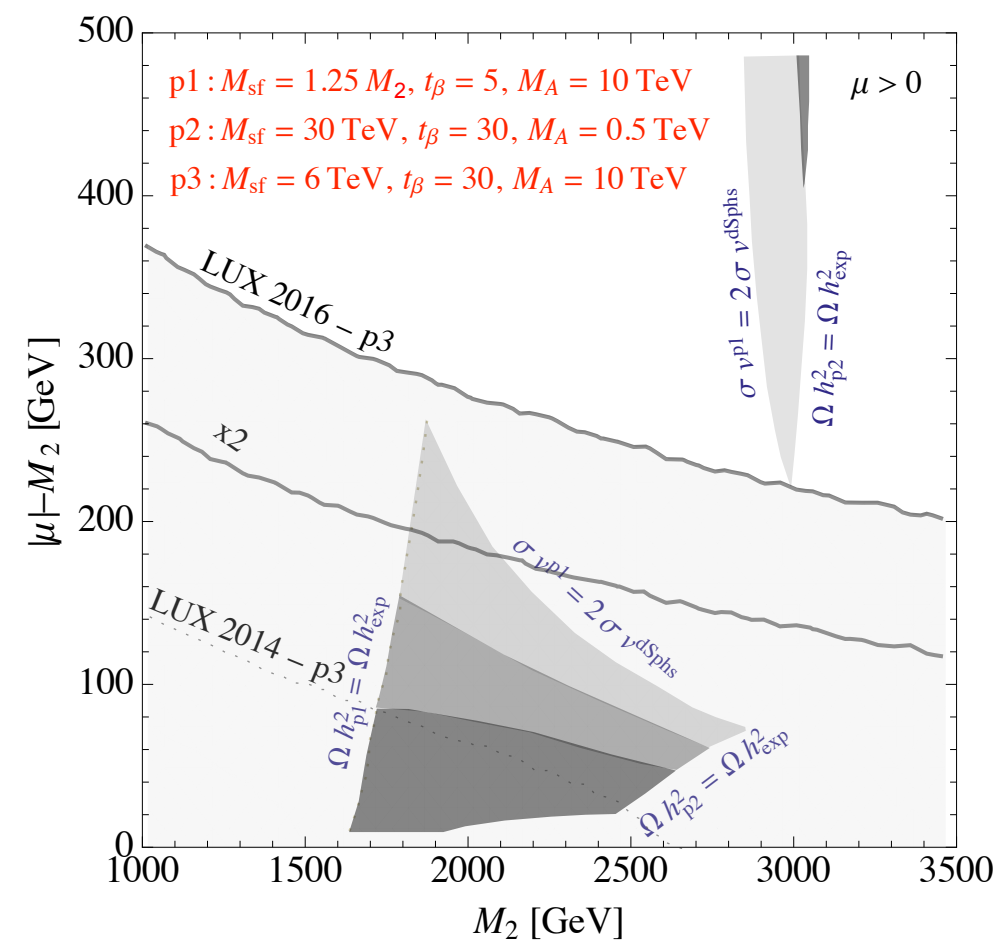

Figure 10. Shaded areas denote the maximal region in the $M_{2}$ vs $|\mu|-M_{2}$ plane for $\mu>0$ where the relic density is as observed and the limit from dSphs diffuse gamma searches is respected within parameter ranges considered. The darker the grey region, the more stringent is the choice of the bound as described in the text. The grey lines mark the weakest possible limit of the region excluded by the 2016 LUX results and the same limit weakened by a factor of two as indicated. The limit from the previous LUX result is the dotted line. The different bounds are calculated at different parameter sets p1, p2 and p3, as indicated.

of the studied parameter space. In particular, the weakest possible limits correspond to $M_{\mathrm{sf}}=1.25 M_{2}, M_{A}=0.5 \mathrm{TeV}$ and $\tan \beta=15$. Note that for $M_{A}=0.5 \mathrm{TeV}$ a significantly lower value of $\tan \beta$ would be in conflict with constraints from heavy Higgs searches at the LHC.

The result of varying $M_{A}, M_{\mathrm{sf}}$ and $\tan \beta$ is a sizeable mass region for viable mixed-wino dark matter in the MSSM, ranging from $M_{2}=1.6$ to $3 \mathrm{TeV}$, as shown in figure 11. The parameter $|\mu|-M_{2}$ for the Higgsino admixture varies from close to $0 \mathrm{GeV}$ to $210 \mathrm{GeV}$ below the Sommerfeld resonance, and from $200 \mathrm{GeV}$ upwards above, when the most conservative dSphs limit (shown in light grey) is adopted.

We note that in determining the viable mixed-wino parameter region we did not include the diffuse gamma-ray and gamma line data from observations of the Galactic center, since the more conservative assumption of a cored dark matter profile would not provide a further constraint. However, future gamma data, in particular CTA observations of the Galactic center, are expected to increase the sensitivity to the parameter region in question to the extent (cf. [5]) that either a dominantly-wino neutralino dark matter would be seen, or the entire plane shown in figure 11 would be excluded even for a cored profile. 


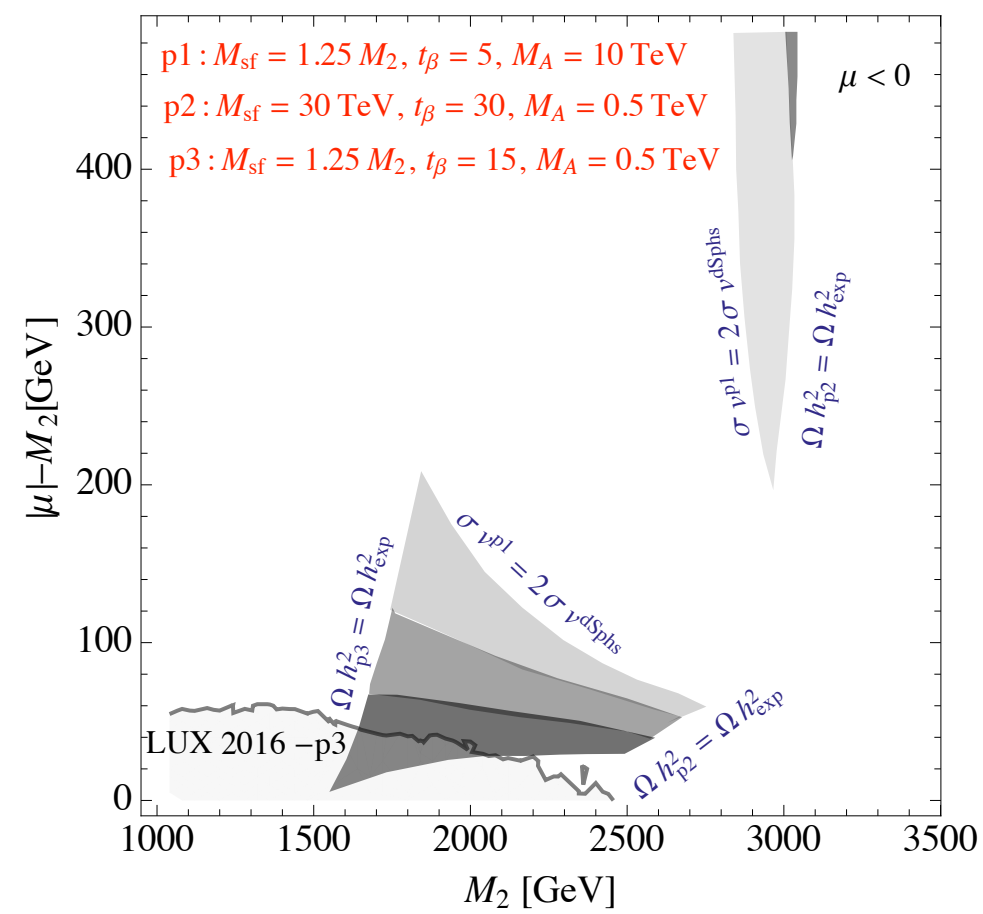

Figure 11. Maximal region in the $M_{2}$ vs $\mu-M_{2}$ plane for $\mu<0$, obtained as in figure 10. The limit from the 2016 LUX result weakened by a factor of two is not visible within the ranges considered in the plot. The different bounds are calculated at different parameter sets $\mathrm{p} 1, \mathrm{p} 2$ and $\mathrm{p} 3$, as indicated.

\section{Conclusions}

This study was motivated by the wish to delineate the allowed parameter (in particular mass) range for a wino-like dark matter particle in the MSSM, only allowing some mixing with the Higgsino. More generically, this corresponds to the case where the dark matter particle is the lightest state of a heavy electroweak triplet with potentially significant doublet admixture and the presence of a scalar mediator. The Sommerfeld effect is always important in the $\mathrm{TeV}$ mass range, where the observed relic density can be attained, and has been included in this study extending previous work $[1,9,10]$. Our main results are summarized in figures 10 and 11, which show the viable parameter region for the dominantly-wino neutralino for the cases $\mu>0$ and $\mu<0$, respectively. After imposing the collider and flavour constraints (both very weak), we considered the limits from diffuse gamma-rays from the dwarf spheroidal galaxies (dSphs), galactic cosmic rays and cosmic microwave background anisotropies. We also calculated the antiproton flux in order to compare with the AMS-02 results. The choice of indirect search constraints is influenced by the attitude that the fundamental question of the viability of wino-like dark matter should be answered by adopting conservative assumptions on astrophysical uncertainties. The non-observation of an excess of diffuse gamma-rays from dSphs then provides the strongest limit. 
It turns out that in addition to these indirect detection bounds, the direct detection results have a significant impact on the parameter space, particularly for the $\mu>0$ case where the mixed Higgsino-wino region is almost ruled out as shown in figure 10. In the $\mu<0$ case the limits are weaker as seen in figure 11, and a sizeable viable region remains. Note that the region of the $|\mu|-M_{2}$ vs. $M_{2}$ plane constrained by direct detection is complementary to that constrained by indirect detection. Therefore while for $\mu>0$, (almost) the entire mixed region is ruled out, for $\mu<0$ there is a part of parameter space where $M_{2}=1.7-2.7 \mathrm{TeV}$ which is in complete agreement with all current experimental constraints.

Let us conclude by commenting on the limits from line and diffuse photon spectra from the Galactic center. If a cusped or mildly cored DM profile was assumed, the H.E.S.S. observations of diffuse gamma emission [53] would exclude nearly the entire parameter space considered in this paper, leaving only a very narrow region with close to maximal wino-Higgsino mixing. The limits from searches for a line-like feature [54] would be even stronger, leaving no space for mixed-wino neutralino DM. However, a cored DM profile remains a possibility, and hence we did not include the H.E.S.S. results. In other words, adopting a less conservative approach, one would conclude that not only the pure-wino limit of the MSSM, but also the entire parameter region of the dominantly-wino neutralino, even with very large Higgsino or bino admixture, was in strong tension with the indirect searches. Therefore, the forthcoming observations by CTA should either discover a signal of or definitively exclude the dominantly-wino neutralino.

\section{Acknowledgments}

We thank A. Ibarra for comments on the manuscript, and A. Goudelis and V. Rentala for helpful discussions. This work is supported in part by the Gottfried Wilhelm Leibniz programme of the Deutsche Forschungsgemeinschaft (DFG) and the Excellence Cluster "Origin and Structure of the Universe" at Technische Universität München. AH is supported by the University of Oslo through the Strategic Dark Matter Initiative (SDI). We further gratefully acknowledge that part of this work was performed using the facilities of the Computational Center for Particle and Astrophysics (C2PAP) of the Excellence Cluster.

Open Access. This article is distributed under the terms of the Creative Commons Attribution License (CC-BY 4.0), which permits any use, distribution and reproduction in any medium, provided the original author(s) and source are credited.

\section{References}

[1] M. Beneke et al., Relic density of wino-like dark matter in the MSSM, JHEP 03 (2016) 119 [arXiv: 1601.04718] [INSPIRE].

[2] A. Hryczuk, I. Cholis, R. Iengo, M. Tavakoli and P. Ullio, Indirect Detection Analysis: Wino Dark Matter Case Study, JCAP 07 (2014) 031 [arXiv:1401.6212] [InSPIRE].

[3] T. Cohen, M. Lisanti, A. Pierce and T.R. Slatyer, Wino Dark Matter Under Siege, JCAP 10 (2013) 061 [arXiv:1307.4082] [INSPIRE]. 
[4] J. Fan and M. Reece, In Wino Veritas? Indirect Searches Shed Light on Neutralino Dark Matter, JHEP 10 (2013) 124 [arXiv:1307.4400] [INSPIRE].

[5] L. Roszkowski, E.M. Sessolo and A.J. Williams, Prospects for dark matter searches in the pMSSM, JHEP 02 (2015) 014 [arXiv:1411.5214] [INSPIRE].

[6] V. Lefranc, E. Moulin, P. Panci, F. Sala and J. Silk, Dark Matter in $\gamma$ lines: Galactic Center vs dwarf galaxies, JCAP 09 (2016) 043 [arXiv: 1608.00786] [INSPIRE].

[7] M. Beneke, C. Hellmann and P. Ruiz-Femenia, Non-relativistic pair annihilation of nearly mass degenerate neutralinos and charginos I. General framework and S-wave annihilation, JHEP 03 (2013) 148 [Erratum ibid. 10 (2013) 224] [arXiv:1210.7928] [INSPIRE].

[8] C. Hellmann and P. Ruiz-Femenía, Non-relativistic pair annihilation of nearly mass degenerate neutralinos and charginos II. P-wave and next-to-next-to-leading order S-wave coefficients, JHEP 08 (2013) 084 [arXiv:1303.0200] [INSPIRE].

[9] M. Beneke, C. Hellmann and P. Ruiz-Femenia, Non-relativistic pair annihilation of nearly mass degenerate neutralinos and charginos III. Computation of the Sommerfeld enhancements, JHEP 05 (2015) 115 [arXiv:1411.6924] [INSPIRE].

[10] M. Beneke, C. Hellmann and P. Ruiz-Femenia, Heavy neutralino relic abundance with Sommerfeld enhancements - a study of pMSSM scenarios, JHEP 03 (2015) 162 [arXiv: 1411.6930] [INSPIRE].

[11] AMS collaboration, M. Aguilar et al., Antiproton Flux, Antiproton-to-Proton Flux Ratio and Properties of Elementary Particle Fluxes in Primary Cosmic Rays Measured with the Alpha Magnetic Spectrometer on the International Space Station, Phys. Rev. Lett. 117 (2016) 091103 [INSPIRE].

[12] M. Ibe, S. Matsumoto, S. Shirai and T.T. Yanagida, Wino Dark Matter in light of the AMS-02 2015 Data, Phys. Rev. D 91 (2015) 111701 [arXiv:1504.05554] [INSPIRE].

[13] LUX collaboration, D.S. Akerib et al., First results from the LUX dark matter experiment at the Sanford Underground Research Facility, Phys. Rev. Lett. 112 (2014) 091303 [arXiv: 1310.8214] [INSPIRE].

[14] D.S. Akerib et al., Results from a search for dark matter in the complete LUX exposure, arXiv: 1608.07648 [INSPIRE].

[15] M. Cirelli et al., PPPC \& DM ID: A Poor Particle Physicist Cookbook for Dark Matter Indirect Detection, JCAP 03 (2011) 051 [Erratum ibid. 10 (2012) E01] [arXiv:1012.4515] [INSPIRE].

[16] P. Ciafaloni, D. Comelli, A. Riotto, F. Sala, A. Strumia and A. Urbano, Weak Corrections are Relevant for Dark Matter Indirect Detection, JCAP 03 (2011) 019 [arXiv:1009.0224] [INSPIRE].

[17] V.S. Berezinsky, S.V. Bulanov, V.A. Dogiel and V.S. Ptuskin, Astrophysics of cosmic rays, V.L. Ginzburg ed., North-Holland, Amsterdam (1990).

[18] C. Evoli, D. Gaggero, D. Grasso and L. Maccione, Cosmic-Ray Nuclei, Antiprotons and Gamma-rays in the Galaxy: a New Diffusion Model, JCAP 10 (2008) 018 [arXiv:0807.4730] [INSPIRE].

[19] M. Tavakoli, Three Dimensional Distribution of Atomic Hydrogen in the Milky Way, arXiv:1207.6150 [INSPIRE].

[20] M. Pohl, P. Englmaier and N. Bissantz, 3D Distribution of Molecular Gas in the Barred Milky Way, Astrophys. J. 677 (2008) 283 [arXiv:0712.4264] [INSPIRE]. 
[21] L.J. Gleeson and W.I. Axford, Solar Modulation of Galactic Cosmic Rays, Astrophys. J. 154 (1968) 1011 [INSPIRE].

[22] F. Donato, N. Fornengo, D. Maurin and P. Salati, Antiprotons in cosmic rays from neutralino annihilation, Phys. Rev. D 69 (2004) 063501 [astro-ph/0306207] [INSPIRE].

[23] A. Oliva, AMS results on light nuclei: Measurement of the cosmic rays boron-to-carbon ration with AMS-02, presentation at AMS Days at CERN - The future of Cosmic Ray Physics and latest results, CERN, Geneva, Switzerland, 15-17 April 2015.

[24] S. Haino, Precision measurement of he flux with AMS, presentation at AMS Days at CERN - The future of Cosmic Ray Physics and latest results, CERN, Geneva, Switzerland, 15-17 April 2015.

[25] AMS collaboration, M. Aguilar et al., Precision Measurement of the Proton Flux in Primary Cosmic Rays from Rigidity $1 G V$ to 1.8 TV with the Alpha Magnetic Spectrometer on the International Space Station, Phys. Rev. Lett. 114 (2015) 171103 [INSPIRE].

[26] PAMELA collaboration, O. Adriani et al., PAMELA Measurements of Cosmic-ray Proton and Helium Spectra, Science 332 (2011) 69 [arXiv:1103.4055] [INSPIRE].

[27] O. Adriani et al., Measurement of boron and carbon fluxes in cosmic rays with the PAMELA experiment, Astrophys. J. 791 (2014) 93 [arXiv:1407.1657] [INSPIRE].

[28] AMS collaboration, L. Accardo et al., High Statistics Measurement of the Positron Fraction in Primary Cosmic Rays of 0.5-500 GeV with the Alpha Magnetic Spectrometer on the International Space Station, Phys. Rev. Lett. 113 (2014) 121101 [InSPIRE].

[29] AMS collaboration, M. Aguilar et al., Precision Measurement of the $\left(e^{+}+e^{-}\right)$Flux in Primary Cosmic Rays from $0.5 \mathrm{GeV}$ to $1 \mathrm{TeV}$ with the Alpha Magnetic Spectrometer on the International Space Station, Phys. Rev. Lett. 113 (2014) 221102 [INSPIRE].

[30] J.J. Engelmann, P. Ferrando, A. Soutoul, P. Goret and E. Juliusson, Charge composition and energy spectra of cosmic-ray for elements from Be to NI - Results from HEAO-3-C2, Astron. Astrophys. 233 (1990) 96 [INSPIRE].

[31] H.S. Ahn et al., Measurements of cosmic-ray secondary nuclei at high energies with the first flight of the CREAM balloon-borne experiment, Astropart. Phys. 30 (2008) 133 [arXiv: 0808.1718] [INSPIRE].

[32] S.P. Swordy, D. Mueller, P. Meyer, J. L'Heureux and J.M. Grunsfeld, Relative abundances of secondary and primary cosmic rays at high energies, Astrophys. J. 349 (1990) 625.

[33] J.S. George et al., Elemental composition and energy spectra of galactic cosmic rays during solar cycle 23, Astrophys. J. 698 (2009) 1666.

[34] R. Kappl, A. Reinert and M.W. Winkler, AMS-02 Antiprotons Reloaded, JCAP 10 (2015) 034 [arXiv: 1506.04145] [INSPIRE].

[35] C. Evoli, D. Gaggero and D. Grasso, Secondary antiprotons as a Galactic Dark Matter probe, JCAP 12 (2015) 039 [arXiv: 1504.05175] [INSPIRE].

[36] G. Giesen et al., AMS-02 antiprotons, at last! Secondary astrophysical component and immediate implications for Dark Matter, JCAP 09 (2015) 023 [arXiv:1504.04276] [INSPIRE].

[37] Fermi-LAT, MAGIC collaborations, M.L. Ahnen et al., Limits to dark matter annihilation cross-section from a combined analysis of MAGIC and Fermi-LAT observations of dwarf satellite galaxies, JCAP 02 (2016) 039 [arXiv:1601.06590] [INSPIRE]. 
[38] N. Padmanabhan and D.P. Finkbeiner, Detecting dark matter annihilation with CMB polarization: Signatures and experimental prospects, Phys. Rev. D 72 (2005) 023508 [astro-ph/0503486] [INSPIRE].

[39] S. Galli, F. Iocco, G. Bertone and A. Melchiorri, CMB constraints on Dark Matter models with large annihilation cross-section, Phys. Rev. D 80 (2009) 023505 [arXiv:0905.0003] [INSPIRE].

[40] T.R. Slatyer, N. Padmanabhan and D.P. Finkbeiner, CMB Constraints on WIMP Annihilation: Energy Absorption During the Recombination Epoch, Phys. Rev. D 80 (2009) 043526 [arXiv: 0906.1197] [INSPIRE].

[41] Planck collaboration, P.A.R. Ade et al., Planck 2015 results. XIII. Cosmological parameters, Astron. Astrophys. 594 (2016) A13 [arXiv:1502.01589] [INSPIRE].

[42] M.S. Madhavacheril, N. Sehgal and T.R. Slatyer, Current Dark Matter Annihilation Constraints from CMB and Low-Redshift Data, Phys. Rev. D 89 (2014) 103508 [arXiv: 1310.3815] [INSPIRE].

[43] J. Hisano, K. Ishiwata, N. Nagata and T. Takesako, Direct Detection of Electroweak-Interacting Dark Matter, JHEP 07 (2011) 005 [arXiv:1104.0228] [INSPIRE].

[44] PandaX-II collaboration, A. Tan et al., Dark Matter Results from First 98.7 Days of Data from the PandaX-II Experiment, Phys. Rev. Lett. 117 (2016) 121303 [arXiv:1607.07400] [INSPIRE].

[45] J. Bovy and S. Tremaine, On the local dark matter density, Astrophys. J. 756 (2012) 89 [arXiv: 1205.4033] [INSPIRE].

[46] S. Dürr et al., Lattice computation of the nucleon scalar quark contents at the physical point, Phys. Rev. Lett. 116 (2016) 172001 [arXiv:1510.08013] [INSPIRE].

[47] G. Bélanger et al., Indirect search for dark matter with MicrOMEGAs2.4, Comput. Phys. Commun. 182 (2011) 842 [arXiv: 1004.1092] [INSPIRE].

[48] G. Bélanger, F. Boudjema, A. Pukhov and A. Semenov, MicrOMEGAs_3: A program for calculating dark matter observables, Comput. Phys. Commun. 185 (2014) 960 [arXiv: 1305.0237] [INSPIRE].

[49] A. Crivellin, M. Hoferichter, M. Procura and L.C. Tunstall, Light stops, blind spots and isospin violation in the MSSM, JHEP 07 (2015) 129 [arXiv: 1503.03478] [INSPIRE].

[50] P. Ullio and M. Valli, A critical reassessment of particle Dark Matter limits from dwarf satellites, JCAP 07 (2016) 025 [arXiv: 1603.07721] [INSPIRE].

[51] A. Kounine, Latest results from the alpha magnetic spectrometer: positron fraction and antiproton/proton ratio, presentation at AMS Days at CERN - The future of Cosmic Ray Physics and latest results, CERN, Geneva, Switzerland, 15-17 April 2015.

[52] O. Adriani et al., Measurement of the flux of primary cosmic ray antiprotons with energies of 60-MeV to 350-GeV in the PAMELA experiment, JETP Lett. 96 (2013) 621 [INSPIRE].

[53] H.E.S.S. collaboration, H. Abdallah et al., Search for dark matter annihilations towards the inner Galactic halo from 10 years of observations with H.E.S.S, Phys. Rev. Lett. 117 (2016) 111301 [arXiv: 1607.08142] [INSPIRE].

[54] H.E.S.S. collaboration, A. Abramowski et al., Search for Photon-Linelike Signatures from Dark Matter Annihilations with H.E.S.S., Phys. Rev. Lett. 110 (2013) 041301 [arXiv: 1301.1173] [INSPIRE]. 